\title{
Embedding subshifts of finite type into the Fibonacci-Dyck shift
}

\author{
TOSHIHIRO HAMACHI $\dagger$ and WOLFGANG KRIEGER $\ddagger$ \\ $\dagger$ Faculty of Mathematics, Kyushu University, 744 Motooka, \\ Nishi-ku, Fukuoka 819-0395, Japan \\ (e-mail: hamachi@math.kyushu-u.ac.jp) \\ $\ddagger$ Institute for Applied Mathematics, University of Heidelberg, \\ Im Neuenheimer Feld 294, 69120 Heidelberg, Germany \\ (e-mail: krieger@math.uni-heidelberg.de)
}

(Received 29 June 2015 and accepted in revised form 16 October 2015)

Abstract. A necessary and sufficient condition is given for the existence of an embedding of an irreducible subshift of finite type into the Fibonacci-Dyck shift.

\section{Introduction}

Let $\Sigma$ be a finite alphabet, and let $S$ be the shift on the shift space $\Sigma^{\mathbb{Z}}$,

$$
S\left(\left(x_{i}\right)_{i \in \mathbb{Z}}\right)=\left(x_{i+1}\right)_{i \in \mathbb{Z}}, \quad\left(x_{i}\right)_{i \in \mathbb{Z}} \in \Sigma^{\mathbb{Z}} .
$$

An $S$-invariant closed subset $X$ of $\Sigma^{\mathbb{Z}}$ is called a subshift. For an introduction to the theory of subshifts see [Ki] or [LM]. A word is called admissible for the subshift $X \subset \Sigma^{\mathbb{Z}}$ if it appears in a point of $X$. A subshift is uniquely determined by its language of admissible words.

Among the first examples of subshifts are the subshifts of finite type. A subshift of finite type is constructed from a finite set $\mathcal{F}$ of words in the alphabet $\Sigma$ as the subshift that contains the points in $\Sigma^{\mathbb{Z}}$ in which no word in $\mathcal{F}$ appears. Other prototypical examples of subshifts are the Dyck shifts. To recall the construction of the Dyck shifts [Kr1], let $N>1$ and let

$$
\alpha_{-}(n), \alpha_{+}(n), \quad 0 \leq n<N,
$$

be the generators of the Dyck inverse monoid $[\mathbf{N P}] \mathcal{D}_{N}$ with the relations

$$
\alpha_{-}(n) \alpha_{+}(m)= \begin{cases}\mathbf{1} & \text { if } n=m, \\ 0 & \text { if } n \neq m .\end{cases}
$$

The Dyck shifts are defined as the subshifts

$$
D_{N} \subset\left(\left\{\alpha_{-}(n): 0 \leq n<N\right\} \cup\left\{\alpha_{+}(n): 0 \leq n<N\right\}\right)^{\mathbb{Z}}
$$


with the admissible words $\left(\sigma_{i}\right)_{1 \leq i \leq I}, I \in \mathbb{N}$, of $D_{N}, N>1$, given by the condition

$$
\prod_{1 \leq i \leq I} \sigma_{i} \neq 0
$$

In $[\mathbf{H I}]$, a necessary and sufficient condition was given for the existence of an embedding of an irreducible subshift of finite type into a Dyck shift. In [HIK], this result was extended to a wider class of target shifts that contains the $\mathcal{D}_{N}$-presentations. With the semigroup $\mathcal{D}_{N}^{-}\left(\mathcal{D}_{N}^{+}\right)$, that is freely generated by $\left\{\alpha_{-}(n): 0 \leq n<N\right\}\left(\left\{\alpha_{+}(n): 0 \leq n<N\right\}\right), \mathcal{D}_{N^{-}}$ presentations can be described as arising from a finite irreducible directed labelled graph with vertex set $\mathcal{V}$, edge set $\Sigma$ and a label map

$$
\lambda: \Sigma \rightarrow \mathcal{D}_{N}^{-} \cup\{\mathbf{1}\} \cup \mathcal{D}_{N}^{+}
$$

that extends to directed paths $b=\left(b_{i}\right)_{1 \leq i \leq I}, I>1$, in the directed graph $(\mathcal{V}, \Sigma)$, by $\lambda(b)=\prod_{1 \leq i \leq I} \lambda\left(b_{i}\right)$. It is required that there exists, for $U, W \in \mathcal{V}$ and for $\beta \in \mathcal{D}_{N}$, a path $b$ from $U$ to $W$ such that $\lambda(b)=\beta$. The $\mathcal{D}_{N}$-presentation $X(\mathcal{V}, \Sigma, \lambda)$ is the subshift with alphabet $\Sigma$ and with the set of admissible words given by the set of directed finite paths $b$ in the graph $(\mathcal{V}, \Sigma, \lambda)$ such that $\lambda(b) \neq 0$.

For Dyck shifts, the notion of a multiplier was introduced in $[\mathbf{H I}]$. A multiplier of the Dyck shift $D_{N}, N \in \mathbb{N}$, or, more generally, of a $\mathcal{D}_{N}$-presentation [HIK], is an equivalence class of primitive words in the symbols $\alpha(n), 0 \leq n \leq N$. Here a word is called primitive if it is not the power of another word, and two primitive words are equivalent if one is a cyclic permutation of the other. The multipliers are the primitive necklaces of combinatorics [BP, $\S 4]$. We use one of its representatives as a notation for a multiplier.

We denote the period of a periodic point $p$ of a $\mathcal{D}_{N}$-presentation $X(\mathcal{V}, \Sigma, \lambda)$ by $\Pi(p)$. A periodic point $p=\left(p_{i}\right)_{i \in \mathbb{Z}}$ of a $\mathcal{D}_{N}$-presentation $X(\mathcal{V}, \Sigma, \lambda)$, and its orbit, are said to be neutral if there exists an $i \in \mathbb{Z}$ such that $\lambda\left(\left(p_{j}\right)_{i \leq j<i+\Pi(p)}\right)=\mathbf{1}$, and they are said to have negative (positive) multiplier if there exists an $i \in \mathbb{Z}$ such that $\lambda\left(\left(p_{j}\right)_{i \leq j<i+\Pi(p)}\right) \in \mathcal{D}_{N}^{-}\left(\mathcal{D}_{N}^{+}\right)$. More precisely, given a multiplier $\mu$, a periodic point $p=\left(p_{i}\right)_{i \in \mathbb{Z}}$ of a $\mathcal{D}_{N}$-presentation $X(\mathcal{V}, \Sigma, \lambda)$, and its orbit, are said to have (negative) multiplier $\mu_{-}$if there exists an $i \in \mathbb{Z}$ and a representative $\left(\alpha\left(n_{j}\right)\right)_{1 \leq j \leq J}$ of $\mu$ such that $\lambda\left(\left(p_{j}\right)_{i \leq j<i+\Pi(p)}\right)$ is equal to $\prod_{1 \leq j \leq J} \alpha_{-}\left(n_{j}\right)$, and are said to have (positive) multiplier $\mu_{+}$if there exists an $i \in \mathbb{Z}$ and a representative $\left(\alpha\left(n_{j}\right)\right)_{1 \leq j \leq J}$ of $\mu$ such that $\lambda\left(\left(p_{j}\right)_{i \leq j<i+\Pi(p)}\right)$ is equal to $\prod_{J \geq j \geq 1} \alpha_{+}\left(n_{j}\right)$. Denote the set of periodic orbits of length $n$ of the $\mathcal{D}_{N}$-presentation $X(\mathcal{V}, \Sigma, \lambda)$ with negative (positive) multiplier by $\mathcal{O}_{n}^{-}\left(X(\mathcal{V}, \Sigma, \lambda)\left(\mathcal{O}_{n}^{+}(X(\mathcal{V}, \Sigma, \lambda))\right.\right.$, and denote the set of its periodic orbits of length $n$ with multiplier $\mu_{-}\left(\mu_{+}\right)$by $\mathcal{O}_{n}\left(\mu_{-}\right)\left(X(\mathcal{V}, \Sigma, \lambda)\left(\mathcal{O}_{n}\left(\mu_{+}\right)(X(\mathcal{V}, \Sigma, \lambda))\right.\right.$. The notion of an exceptional multiplier was introduced in [HI]. A multiplier $\mu$ of a $\mathcal{D}_{N}$-presentation $X(\mathcal{V}, \Sigma, \lambda)$ is said to be exceptional at period $n \in \mathbb{N}$ if

$$
\begin{aligned}
\operatorname{card}\left(\mathcal{O}_{n}\left(\mu_{-}\right)(X(\mathcal{V}, \Sigma, \lambda))\right. & \left.\cup \mathcal{O}_{n}\left(\mu_{+}\right)(X(\mathcal{V}, \Sigma, \lambda))\right) \\
& >\max \left\{\operatorname{card}\left(\mathcal{O}_{n}^{-}(X(\mathcal{V}, \Sigma, \lambda))\right), \operatorname{card}\left(\mathcal{O}_{n}^{+}(X(\mathcal{V}, \Sigma, \lambda))\right)\right\}
\end{aligned}
$$

The Fibonacci-Dyck shift is the Markov-Dyck shift $[\mathbf{M}]$ of the Fibonacci graph. We introduce the Fibonacci graph as the directed graph with vertex set $\{0,1\}$ and edge set $\{\beta(0), \beta, \beta(1)\}: \beta(0)$ is a loop at vertex 0 , the edge $\beta(1)$ goes from vertex 1 to vertex 0 
and the edge $\beta$ from vertex 0 to vertex 1 . Let $\left(\{0,1\},\left\{\beta^{-}(0), \beta^{-}, \beta^{-}(1)\right\}\right)$ be a copy of the Fibonacci graph, and let $\left(\{0,1\},\left\{\beta^{+}(0), \beta^{+}, \beta^{+}(1)\right\}\right)$ be its reversal. The FibonacciDyck shift is given by a $\mathcal{D}_{2}$-presentation, with

$$
\mathcal{V}=\{0,1\}, \quad \Sigma=\left\{\beta^{-}(0), \beta^{-}, \beta^{-}(1), \beta^{+}(0), \beta^{+}, \beta^{+}(1)\right\}
$$

and

$$
\lambda: \Sigma \rightarrow\left\{\alpha_{-}(0), \alpha_{-}(1), \alpha_{+}(0), \alpha_{+}(1)\right\}
$$

given by

$$
\begin{gathered}
\lambda\left(\beta^{-}(0)\right)=\alpha_{-}(0), \quad \lambda\left(\beta^{+}(0)\right)=\alpha_{+}(0), \\
\lambda\left(\beta^{-}(1)\right)=\alpha_{-}(1), \quad \lambda\left(\beta^{+}(1)\right)=\alpha_{+}(1), \\
\lambda\left(\beta_{-}\right)=\lambda\left(\beta_{+}\right)=\mathbf{1} .
\end{gathered}
$$

For this $\mathcal{D}_{2}$-presentation we choose a label map $\lambda$ that assigns the label $\mathbf{1}$ to the edges $\beta^{-}$ and $\beta^{+}$, since these edges arise from the splitting of the edge $\beta$ in the Fibonacci graph. The edge $\beta$ is contracted to a vertex in the procedure that turns the Fibonacci graph into the 1 -vertex graph with two loops, whose graph inverse semigroup is $\mathcal{D}_{2}$. (For this part of the theory see [Kr2, HK]. In [HK, §2], it is shown that the Fibonacci-Dyck shift has Property (A) and, in [HK, §3], it is shown that its associated semigroup is $\mathcal{D}_{2}$. The procedure is also described in [HK, §3].) In this paper we consider, exclusively, the Fibonacci-Dyck shift $F$.

The label map $\lambda$ can be written in the form of a matrix with entries in the semigroup ring of $\mathcal{D}_{2}$, that is

$$
\left(\begin{array}{cc}
a^{-}(0)+a^{+}(0) & \mathbf{1}+a^{+}(1) \\
\mathbf{1}+a^{-}(1) & 0
\end{array}\right) .
$$

Taking the adjoint and applying the involution of the semigroup ring of $\mathcal{D}_{2}$ to its entries, does not change the matrix. This symmetry property of the matrix makes visible the time reversal $\rho$ of $F$ that is obtained by setting

$$
\begin{aligned}
\chi\left(\beta^{-}\right) & =\beta^{+}, \quad \chi\left(\beta^{+}\right)=\beta^{-}, \\
\chi\left(\beta^{-}(0)\right) & =\beta^{+}(0), \chi\left(\beta^{+}(0)\right)=\beta^{-}(0), \\
\chi\left(\beta^{-}(1)\right) & =\beta^{+}(1), \chi\left(\beta^{+}(1)\right)=\beta^{-}(1),
\end{aligned}
$$

and

$$
\rho\left(\left(x_{i}\right)_{i \in \mathbb{Z}}\right)=\left(\chi\left(x_{-i}\right)\right)_{i \in \mathbb{Z}}, \quad x \in F .
$$

We denote the set of multipliers of $F$ by $\mathcal{M}$. The time reversal $\rho$ maps the set $\mathcal{O}_{n}\left(\mu_{-}\right)$ bijectively onto the set $\mathcal{O}_{n}\left(\mu_{+}\right)$and we can note the following lemma.

LEMMA (a).

$$
\operatorname{card}\left(\mathcal{O}_{n}\left(\mu_{-}\right)\right)=\operatorname{card}\left(\mathcal{O}_{n}\left(\mu_{+}\right)\right), \quad n \in \mathbb{N}, \mu \in \mathcal{M}
$$

We also note orbit counts of the Fibonacci-Dyck shift for small periods as a lemma. 
LEMMA (b).

$$
\begin{aligned}
\operatorname{card}\left(\mathcal{O}_{3}\left(\alpha^{-}(0)\right)\right) & =2, \\
\operatorname{card}\left(\mathcal{O}_{5}\left(\alpha^{-}(0)\right)\right) & =9, \\
\operatorname{card}\left(\mathcal{O}_{5}\left(\alpha^{-}(0) \alpha^{-}(1)\right)\right) & =5 . \\
\operatorname{card}\left(\mathcal{O}_{4}\left(\alpha^{-}(0)\right)\right) & =2, \\
\operatorname{card}\left(\mathcal{O}_{4}\left(\alpha^{-}(1)\right)\right) & =3, \\
\operatorname{card}\left(\mathcal{O}_{6}\left(\alpha^{-}(0)\right)\right) & =10, \\
\operatorname{card}\left(\mathcal{O}_{6}\left(\alpha^{-}(1)\right)\right) & =13 .
\end{aligned}
$$

We note a consequence of Lemma (a), also as a lemma.

Lemma (c). A multiplier $\mu \in \mathcal{M}$ is exceptional at period $n \in \mathbb{N}$ if and only if

$$
\operatorname{card}\left(\mathcal{O}_{n}\left(\mu_{-}\right)\right)>\operatorname{card}\left(\bigcup_{\tilde{\mu} \in \mathcal{M} \backslash\{\mu\}} \mathcal{O}_{n}\left(\tilde{\mu}_{-}\right)\right) .
$$

The Fibonacci-Dyck shift has the exceptional multiplier $\alpha(0)$ that is exceptional at period one (and, in view of Lemma (b), also at period three), and the exceptional multiplier $\alpha(1)$ that is exceptional at period two. After introducing notation and terminology in $\S 2$, we show in $\S 3$ that the multiplier $\alpha(0) \alpha(1)$ is not exceptional. In $\S 4$, we prove that the remaining multipliers are not exceptional. Based on these results, and on the results of [HIK], we give, in $\S 5$, a necessary and sufficient condition for the existence of an embedding of an irreducible subshift of finite type into the Fibonacci-Dyck shift. The multipliers (especially the exceptional multiplier) enter here in an essential way. Moreover, we show in $\S 6$ that the multiplier $\alpha(0)$ is exceptional only at periods one and three, and we show in $\S 7$ that the multiplier $\alpha(1)$ is exceptional only at period two.

We denote the set of periodic points $p \in F$ with smallest period $n \in \mathbb{N}$ by $P_{n}^{\circ}$, and we denote the set of points $p \in P_{n}^{\circ}$ with negative multiplier $\mu \in \mathcal{M}$ by $P_{n}^{\circ}\left(\mu_{-}\right)$. In the proofs of $\S \S 3,4,6$ and 7 we construct, for the multiplier $\mu$ in question and for a suitably chosen period $k \in \mathbb{N}$, shift commuting injections

$$
\eta_{n}: P_{n}^{\circ}\left(\mu_{-}\right) \rightarrow \bigcup_{\tilde{\mu} \in \mathcal{M} \backslash\{\mu\}} P_{n}^{\circ}\left(\tilde{\mu}_{-}\right), \quad n>k .
$$

We do this by first constructing a partition of $P_{n}^{\circ}(\mu), n>k$, (some sets of which may be empty), together with a shift commuting injection of each set of the partitions into $\bigcup_{\tilde{\mu} \in \mathcal{M} \backslash\{\mu\}} P_{n}^{\circ}\left(\tilde{\mu}_{-}\right)$, where we show injectivity on each set by describing how a point can be reconstructed from its image under $\eta_{n}$. Then we show that the images under $\eta_{n}$ of the sets of the partition are disjoint.

\section{Preliminaries}

We denote the set of admissible words of the Fibonacci-Dyck shift by $\mathcal{L}$. We denote the empty word by $\epsilon$, and the length of a word by $\ell$.

We denote by $\mathcal{C}(0)$ the circular code of words $c=\left(c_{i}\right)_{1 \leq i \leq I} \in \mathcal{L}, I>1$, that begin with the symbol $\beta^{-}(0)$ and are such that $\lambda(c)=\mathbf{1}$, and there is no index $J, 1<J<I$, for 
which

$$
\prod_{1 \leq j \leq J} \lambda\left(c_{j}\right)=\mathbf{1}
$$

Also, we denote by $\mathcal{C}(1)$ the circular code [BPR, §7] of words $\left.c=\left(c_{i}\right)_{1 \leq i \leq I} \in \mathcal{L}\right), I>3$ that begin with the symbol $\beta^{-}$and end with the word $\beta^{+}(1) \beta^{+}$and are such that $\lambda(c)=\mathbf{1}$ and there is no index $J, 1<J<I$, for which $c_{J}=\beta^{+}$and

$$
\prod_{1 \leq j \leq J} \lambda\left(c_{j}\right)=\mathbf{1} \text {. }
$$

We also have the circular codes

$$
\mathcal{C}=\mathcal{C}(0) \cup\left\{\beta^{-} \beta^{+}\right\} \cup \mathcal{C}(1)
$$

and

$$
\mathcal{C}^{\circ}(1)=\beta^{-}(1) \mathcal{C}^{*} \beta^{+}(1)
$$

Note that

$$
\mathcal{C}(0)=\beta^{-}(0) \mathcal{C}^{*} \beta^{+}(0), \quad \mathcal{C}(1)=\beta^{-}\left(\mathcal{C}^{\circ}(1)^{*} \backslash\{\epsilon\}\right) \beta^{+} .
$$

A bijection

$$
\Psi_{\circ}: \mathcal{C}^{\circ}(1) \rightarrow \mathcal{C}(0)
$$

is given by

$$
\Psi_{\circ}\left(\beta^{-}(1) f \beta^{+}(1)\right)=\beta^{-}(0) f \beta^{+}(0), \quad f \in \mathcal{C}^{*} .
$$

The bijection $\Psi_{\circ}: \mathcal{C}^{\circ}(1) \rightarrow \mathcal{C}(0)$ extends to a bijection

$$
\Psi: \mathcal{C}^{\circ}(1)^{*} \rightarrow \mathcal{C}(0)^{*}
$$

by

$$
\Psi\left(\left(c_{k}^{\circ}\right)_{1 \leq k \leq K}\right)=\left(\left(\Psi_{\circ}\left(c_{k}^{\circ}\right)\right)_{1 \leq k \leq K}, \quad c_{k}^{\circ} \in \mathcal{C}^{\circ}(1), 1 \leq k \leq K, K \in \mathbb{N} .\right.
$$

We set

$$
\mathcal{B}(1)=\beta^{-} \mathcal{C}^{\circ}(1)^{*} \beta^{-}(1)
$$

and we define a bijection

$$
\Xi: \mathcal{B}(1) \rightarrow \mathcal{C}(0)
$$

by

$$
\Xi\left(\beta^{-} f^{\circ} \beta^{-}(1)\right)=\beta^{-}(0) \Psi\left(f^{\circ}\right) \beta^{+}(0), \quad f^{\circ} \in \mathcal{C}^{\circ}(1)^{*} .
$$

We also set

$$
\mathcal{B}(0,0)=\beta^{-}(0) \mathcal{C}^{*} \beta^{-}(0),
$$

and we define a bijection

$$
\Phi_{0}: \mathcal{C}(0) \rightarrow \mathcal{B}(0,0)
$$

by

$$
\Phi_{0}\left(\beta^{-}(0) f \beta^{+}(0)\right)=\beta^{-}(0) f \beta^{-}(0), \quad f \in \mathcal{C}^{*} .
$$

We also set

$$
\mathcal{B}(1,1)=\mathcal{B}(1) \mathcal{C}^{*} \beta^{-} \beta^{-}(1),
$$


and we define a bijection

$$
\Phi_{1}: \mathcal{C}(1) \rightarrow \mathcal{B}(1,1)
$$

by

$$
\Phi_{1}\left(\beta^{-} f^{\circ} \beta^{-}(1) f \beta^{+}(1) \beta^{+}\right)=\beta^{-} f^{\circ} \beta^{-}(1) f \beta^{-} \beta^{-}(1), \quad f^{\circ} \in \mathcal{C}^{\circ}(1)^{*}, f \in \mathcal{C}^{*} .
$$

We set

$$
\mathcal{Q}_{0}=\left(\mathcal{C}(0) \cup\left\{\beta^{-} \beta^{+}\right\}\right)^{*} \backslash\left\{\beta^{-} \beta^{+}\right\}^{*},
$$

and we define an injection

$$
\Delta_{0}: \mathcal{Q}_{0} \rightarrow \mathcal{L}
$$

For this we let $f \in \mathcal{Q}_{0}$,

$$
f=\left(c_{k}\right)_{1 \leq k \leq K}, \quad c_{k} \in \mathcal{C}(0) \cup\left\{\beta^{-} \beta^{+}\right\}, 1 \leq k \leq K, K \in \mathbb{N},
$$

set

$$
k_{\circ}(f)=\max \left\{k \in[1, K]: c_{k} \in \mathcal{C}(0)\right\},
$$

and set

$$
\Delta_{0}(f)=\left(\left(c_{k}\right)_{1 \leq k<k_{\circ}(f)}, \Phi_{0}\left(c_{k_{\circ}(f)}\right),\left(c_{k}\right)_{k_{\circ}(f)<k \leq K}\right) .
$$

We also set

$$
\mathcal{Q}_{1}=\mathcal{C}^{*} \backslash\left(\mathcal{C}(0) \cup\left\{\beta^{-} \beta^{+}\right\}\right)^{*},
$$

and define an injection

$$
\Delta_{1}: \mathcal{Q}_{1} \rightarrow \mathcal{L}
$$

For this we let $f \in \mathcal{Q}_{1}$,

$$
f=\left(c_{k}\right)_{1 \leq k \leq K}, \quad c_{k} \in \mathcal{C}, 1 \leq k \leq K, K>1,
$$

set

$$
k_{\circ}(f)=\max \left\{k \in[1, K]: c_{k} \in \mathcal{C}(1)\right\},
$$

and set

$$
\Delta_{1}(f)=\left(\left(c_{k}\right)_{1 \leq k<k_{\circ}(f)}, \quad \Phi_{1}\left(c_{k_{\circ}(f)}\right),\left(c_{k}\right)_{k_{\circ}(f)<k \leq K}\right) .
$$

We put a linear order on the alphabet of $F$. The resulting lexicographic order on $\mathcal{L}$ will be used to single out an element of $\mathbb{Z} / n \mathbb{Z}$ when constructing the shift commuting maps

$$
\eta_{n}: P_{n}^{\circ}\left(\mu_{-}\right) \rightarrow \bigcup_{\tilde{\mu} \in \mathcal{M} \backslash\{\mu\}} P_{n}^{\circ}\left(\tilde{\mu}_{-}\right), \quad n>k .
$$

If a word appears in a point $p \in P_{n}^{\circ}$ with its last symbol at index $i \in \mathbb{Z}$, then we say that the word appears at index $i$. For $p \in F$, we denote by $\mathcal{I}^{(0)}(p)\left(\mathcal{I}^{(1)}(p)\right)$ the set of indices $i \in \mathbb{Z}$ such that $p_{i}=\beta_{-}(0)\left(\beta_{-}(1)\right)$ and

$$
\lambda\left(p_{[i, i+k]}\right) \neq \mathbf{1}, \quad k \in \mathbb{N} .
$$

We say that a word appears openly in $p \in F$ if it appears at an index $i \in \mathcal{I}^{(0)}(p) \cup \mathcal{I}^{(1)}(p)$.

For an element $\gamma$ of the free monoid that is generated by $\alpha(0)$ and $\alpha(1)$ (or by $\alpha_{-}(0)$ and $\left.\alpha_{-}(1)\right)$, for example, for

$$
\gamma=\prod_{0 \leq n<N} \alpha(0)^{K(0, n)} \alpha(1)^{K(1, n)}, \quad K(0, n), K(1, n) \in \mathbb{Z}_{+}, 0 \leq n<N,
$$


we use the notation

$$
v_{0}(\gamma)=\sum_{0 \leq n<N} K(0, n), \quad v_{1}(\gamma)=\sum_{0 \leq n<N} K(1, n),
$$

and, choosing for $\gamma$ any representative of the multiplier $\mu \in \mathcal{M}$, we set

$$
v_{0}(\mu)=v_{0}(\gamma), \quad v_{1}(\mu)=v_{1}(\gamma) .
$$

A point $p \in P_{n}^{\circ}\left(\mu_{-}\right), \mu \in \mathcal{M}$, determines a $\kappa_{p} \in \mathbb{N}$ by

$$
\left(v_{0}\left(\lambda\left(p_{[0, n)}\right)\right), v_{1}\left(\lambda\left(p_{[0, n)}\right)\right)\right)=\kappa_{p}\left(v_{0}(\mu), v_{1}(\mu)\right) .
$$

For

$$
b=\beta^{-} f^{\circ} \beta^{-}(1) \in \mathcal{B}(1),
$$

we set

$$
\Lambda(b)=\ell\left(f^{\circ}\right)
$$

and, for

$$
b=\beta^{-}(0) f \beta^{-}(0) \in \mathcal{B}(0,0),
$$

we set

$$
\Lambda(b)=\ell(f) .
$$

We also define a subset $\mathcal{D}(1,1)$ of $\mathcal{B}(1) \mathcal{C}^{*} \mathcal{B}(1)$ by

$$
\begin{aligned}
\mathcal{D}(1,1)= & \left\{\beta^{-} f^{\circ,-} \beta^{-}(1) f \beta^{-} f^{\circ,+} \beta^{-}(1) \in \beta^{-} \mathcal{C}^{\circ}(1)^{*} \beta^{-}(1) \mathcal{C}^{*} \beta^{-}(0) \mathcal{C}^{\circ}(1)^{*} \beta^{-}(1):\right. \\
& \left.\ell(f) \geq \ell\left(f^{\circ,-}\right), \ell\left(f^{\circ,+}\right)\right\},
\end{aligned}
$$

and a subset $\mathcal{D}(0,1)$ of $\beta^{-}(0) \mathcal{C}^{*} \mathcal{B}(1)$ by

$$
\mathcal{D}(0,1)=\left\{\beta^{-}(0) f \beta^{-} f^{\circ} \beta^{-}(1) \in \beta^{-}(0) \mathcal{C}^{*} \beta^{-} \mathcal{C}^{\circ}(1)^{*} \beta^{-}(1): \ell(f) \geq \ell\left(f^{\circ}\right)\right\},
$$

as well as a subset $\mathcal{D}(1,0)$ of $\mathcal{B}(1) \mathcal{C}^{*} \beta^{-}(0)$ by

$$
\mathcal{D}(1,0)=\left\{\beta^{-} f^{\circ} \beta^{-}(1) f \beta^{-}(0) \in \beta^{-} \mathcal{C}^{\circ}(1)^{*} \beta^{-}(1) \mathcal{C}^{*} \beta^{-}(0): \ell(f) \geq \ell\left(f^{\circ}\right)\right\},
$$

and, for

$$
\begin{gathered}
d=\beta^{-} f^{\circ,-} \beta^{-}(1) f \beta^{-} f^{\circ,+} \beta^{-}(1) \in \mathcal{D}(1,1), \\
d=\beta^{-}(0) f \beta^{-} f^{\circ} \beta^{-}(1) \in \mathcal{D}(0,1),
\end{gathered}
$$

and

$$
d=\beta^{-} f^{\circ} \beta^{-}(1) f \beta^{-}(0) \in \mathcal{D}(1,0),
$$

we set

$$
\Lambda(d)=\ell(f) .
$$

For a point $p \in P_{n}^{\circ}(F)$, we denote by $\Lambda(p)$ the maximal value of $\Lambda(d)$ of words

$$
d \in \mathcal{B}(0,0) \cup \mathcal{B}(1) \cup \mathcal{D}(1,1) \cup \mathcal{D}(0,1) \cup \mathcal{D}(1,0)
$$

that appear openly in $p$, and we denote by $\mathcal{J}^{(0,0)}(p)\left(\mathcal{J}^{(1)}(p), \mathcal{J}^{(1,1)}(p), \mathcal{J}^{(0,1)}(p)\right.$, $\mathcal{J}^{(1,0)}(p)$ the set of indices at which there appears openly in $p$ a word $d \in \mathcal{B}(0,0)$ $(d \in \mathcal{B}(1), d \in \mathcal{D}(1,1), d \in \mathcal{D}(0,1), d \in \mathcal{D}(1,0))$ such that $\Lambda(p)=\Lambda(d)$. Also, we denote by $\mathcal{J}_{\circ}^{(0,0)}(p) \quad\left(\mathcal{J}_{\circ}^{(1)}(p), \mathcal{J}_{\circ}^{(1,1)}(p), \mathcal{J}_{\circ}^{(0,1)}(p), \mathcal{J}_{\circ}^{(1,0)}(p)\right)$ the set of indices $j_{\circ} \in \mathcal{J}^{(0,0)}(p)\left(\mathcal{J}^{(1)}(p), \mathcal{J}^{(1,1)}(p), \mathcal{J}^{(0,1)}(p), \mathcal{J}^{(1,0)}(p)\right)$ such that the word $p_{\left(j_{0}-n, j_{\circ}\right]}$ is lexicographically the smallest one among the words $p_{(j-n, j]}, j \in \mathcal{J}^{(0,0)}(p)\left(\mathcal{J}^{(1)}(p)\right.$, $\left.\mathcal{J}^{(1,1)}(p), \mathcal{J}^{(0,1)}(p), \mathcal{J}^{(1,0)}(p)\right)$. 


\section{The multiplier $\alpha(0) \alpha(1)$}

LEMMA 1. The multiplier $\alpha(0) \alpha(1)$ is not exceptional for the Fibonacci-Dyck shift.

Proof. By Lemma (b), the multiplier $\alpha(0) \alpha(1)$ is not exceptional for the Fibonacci-Dyck shift for periods three and five.

We construct shift commuting injections

$$
\eta_{n}: P_{n}^{\circ}\left(\alpha_{-}(0) \alpha_{-}(1)\right) \rightarrow \bigcup_{\tilde{\mu} \in \mathcal{M} \backslash\{\alpha(0) \alpha(1)\}} P_{n}^{\circ}\left(\tilde{\mu}_{-}\right), \quad n>5 .
$$

Let $m>2$. Let $P_{2 m+1}^{(0)}$ be the set of $p \in P_{2 m+1}^{\circ}\left(\alpha_{-}(0) \alpha_{-}(1)\right)$ such that $\kappa_{p}=1$, which means that

$$
p_{(i-2 m-1, i]} \in \mathcal{C}^{*} \beta^{-}(0) \mathcal{C}^{*} \beta^{-} \mathcal{C}^{\circ}(1)^{*} \beta^{-}(1), \quad i \in \mathcal{I}^{(1)}(p),
$$

and, for $p \in P_{2 m+1}^{(0)}$, let the words

$$
f^{-}(p), f^{+}(p) \in \mathcal{C}^{*}, \quad f^{\circ}(p) \in \mathcal{C}^{\circ}(1)^{*},
$$

be given by writing

$$
p_{(i-2 m-1, i]}=f^{-}(p) \beta^{-}(0) f^{+}(p) \beta^{-} f^{\circ}(p) \beta^{-}(1), \quad i \in \mathcal{I}^{(1)}(p) .
$$

We set

$$
P_{2 m+1}^{[1]}=\left\{p \in P_{2 m+1}^{(0)}: f^{+}(p) \in \mathcal{Q}_{1}\right\} .
$$

The shift commuting map $\eta_{2 m+1}$ is to map a point $p \in P_{2 m+1}^{[1]}$ to the point $q \in P_{2 m+1}^{\circ}$ that is given by

$$
q_{(i-2 m-1, i]}=f^{-}(p) \beta^{-}(0) \Delta_{1}\left(f^{+}(p)\right) \beta^{-} f^{\circ}(p) \beta^{-}(1), \quad i \in \mathcal{I}^{(1)}(p) .
$$

For $q \in \eta_{2 m+1}\left(P_{2 m+1}^{[1]}\right)$,

$$
q_{(i-2 m-1, i]} \in \mathcal{C}^{*} \mathcal{B}(1,1) \mathcal{C}^{*} \mathcal{B}(1) \mathcal{C}^{*} \beta^{-}(0), \quad i \in \mathcal{I}^{(0)}(q),
$$

and, with the words

$$
g(q), g^{-}(q) \in \mathcal{C}^{*}, g^{+}(q) \in\left(\mathcal{C}(0) \cup\left\{\beta^{-} \beta^{+}\right\}\right)^{*}, \quad b(q) \in \mathcal{B}(1,1), h(q) \in \mathcal{B}(1)
$$

that are given by

$$
q_{(i-2 m-1, i]}=g^{-}(q) b(q) g^{+}(q) h(q) g(q) \beta^{-}(0), \quad i \in \mathcal{I}^{(0)}(q),
$$

the point $p \in P_{2 m+1}^{[0]}$ can be reconstructed from its image $q$ under $\eta_{2 m+1}$ as the point in $P_{2 m+1}^{(0)}$ that is given by

$$
p_{(i-2 m-1, i]}=g^{-}(q) \Phi_{1}^{-1}(b(q)) g^{+}(q) h(q) g(q) \beta^{-}(0), \quad i \in \mathcal{I}^{(0)}(q) .
$$

We note that

$\nu_{0}\left(\lambda\left(\eta_{2 m+1}(p)_{[0,2 m+1)}\right)\right)=1, \quad \nu_{1}\left(\lambda\left(\eta_{2 m+1}(p)_{[0,2 m+1)}\right)\right)=3, \quad p \in P_{2 m+1}^{[1]} . \quad(\mathrm{P} .01[1])$

We set

$$
P_{2 m+1}^{[0]}=\left\{p \in P_{2 m+1}^{(0)}: f^{+}(p) \in \mathcal{Q}_{0}\right\} .
$$


The shift commuting map $\eta_{2 m+1}$ is to map a point $p \in P_{2 m+1}^{[0]}$ to the point $q \in P_{2 m+1}^{\circ}(F)$ that is given by

$$
q_{(i-2 m-1, i]}=f^{-}(p) \beta^{-}(0) \Delta_{0}\left(f^{+}(p)\right) \beta^{-} f^{\circ}(p) \beta^{-}(1), \quad i \in \mathcal{I}^{(1)}(p) .
$$

For $q \in \eta_{2 m+1}\left(P_{2 m+1}^{[0]}\right)$,

$$
q_{(i-2 m-1, i]} \in \mathcal{C}^{*} \beta^{-}(0) \mathcal{B}(0,0) \mathcal{C}^{*} \beta^{-} \mathcal{C}^{\circ}(1)^{*} \beta^{-}(1), \quad i \in \mathcal{I}^{(1)}(q) .
$$

and, with the words

$$
\begin{gathered}
\left.g(q) \in \mathcal{C}^{*}, g^{-}(q) \in\left(\mathcal{C}(0) \cup\left\{\beta^{-} \beta^{+}\right\}\right)^{*}, g^{+}(q) \in\left\{\beta^{-} \beta^{+}\right\}\right)^{*}, \\
b(q) \in \mathcal{B}(0,0), g^{\circ}(q) \in \mathcal{C}^{\circ}(1)^{*}
\end{gathered}
$$

that are given by

$$
q_{(i-2 m-1, i]}=g(q) \beta^{-}(0) g^{-}(q) b(q) g^{+}(q) \beta^{-} g^{\circ}(q) \beta^{-}(1), \quad i \in \mathcal{I}^{(1)}(q),
$$

the point $p \in P_{2 m+1}^{[0]}$ can be reconstructed from its image $q$ under $\eta_{2 m+1}$ as the point in $P_{2 m+1}^{(0)}$ that is given by

$$
p_{(i-2 m-1, i]}=g(q) \beta^{-}(0) g^{-}(q) \Phi_{0}^{-1}(b(q)) g^{+}(q) \beta^{-} g^{\circ}(q) \beta^{-}(1), \quad i \in \mathcal{I}^{(1)}(q) .
$$

We note that

$\nu_{0}\left(\lambda\left(\eta_{2 m+1}(p)_{[0,2 m+1)}\right)\right)=3, \quad \nu_{1}\left(\lambda\left(\eta_{2 m+1}(p)_{[0,2 m+1)}\right)\right)=1, \quad p \in P_{2 m+1}^{[1]} . \quad(\mathrm{P} .01[0])$

We set

$$
P_{2 m+1}^{[\beta]}=P_{2 m+1}^{(0)} \backslash\left(P_{2 m+1}^{[1]} \cup P_{2 m+1}^{[0]}\right) .
$$

The shift commuting map $\eta_{2 m+1}$ is to map a point $p \in P_{2 m+1}^{[\beta]}$ to the point $q \in P_{2 m+1}^{\circ}(F)$ that is given by

$$
q_{(i-2 m-1, i]}=f^{-}(p) \beta^{-}(0) f^{+}(p) \Xi\left(\beta^{-} f^{\circ}(p) \beta^{-}(1)\right), \quad i \in \mathcal{I}^{(1)}(p) .
$$

With words

$$
g^{(\beta)}(q) \in\left\{\beta^{-} \beta^{+}\right\}^{*}, \quad c(q) \in \mathcal{C}(0), \quad g(q) \in \mathcal{C}^{*}
$$

that are given by

$$
q_{(i-2 m-1, i]}=g^{(\beta)}(q) c(q) g(q) \beta^{-}(0), \quad i \in \mathcal{I}^{(0)}(q),
$$

a point $p \in P_{2 m+1}^{[\beta]}$ can be reconstructed from its image $q$ under $\eta_{2 m+1}$ as the point $p \in$ $P_{2 m+1}^{(\circ)}$ that is given by

$$
p_{(i-2 m-1, i]}=g^{(\beta)}(q) \Xi^{-1}(c(q)) g(q) \beta^{-}(0), \quad i \in \mathcal{I}^{(0)}(q) .
$$

We note that

$\nu_{0}\left(\lambda\left(\eta_{2 m+1}(p)_{[0,2 m+1)}\right)\right)=1, \quad \nu_{1}\left(\lambda\left(\eta_{2 m+1}(p)_{[0,2 m+1)}\right)\right)=0, \quad p \in P_{2 m+1}^{[\beta]} .(\mathrm{P} .01[\beta])$ 
We set

$$
P_{2 m}^{(0)}=\emptyset, \quad m>3
$$

and, for $n>5$, we set

$$
P_{n}^{(1)}=\left\{p \in P_{n}^{\circ}\left(\alpha_{-}(0) \alpha_{-}(1)\right) \backslash P_{n}^{(0)}: \mathcal{J}^{(1)}(p) \neq \emptyset\right\} .
$$

The shift commuting map $\eta_{n}$ is to map a point $p \in P_{n}^{(1)}$ to the point $q \in P_{n}(F)$ that is obtained by replacing, in the point $p$, each of the words $b \in \mathcal{B}(1)$, that appear at the indices in $\mathcal{J}_{\circ}^{(1)}(p)$, by the word $\Xi(b)$.

A point $p \in P_{n}^{(1)}$ can be reconstructed from its image $q$ under $\eta_{n}$ by replacing, in $q$, the word $c(q) \in \mathcal{C}(0)$, that is identified as the unique word in $\mathcal{C}^{*}$ of maximal length that appears in $q$, by the word $\Xi^{-1}(c(q))$.

We note that

$$
\left(v _ { 0 } \left(\lambda\left(\eta_{n}(p)_{[0, n)}\right), v_{1}\left(\lambda\left(\eta_{n}(p)_{[0, n)}\right)\right)=\left(\kappa_{p}, \kappa_{p}-1\right), \quad p \in P_{n}^{(1)} .\right.\right.
$$

We set

$$
P_{n}^{(0,1)}=\left\{p \in P_{n}^{\circ}\left(\alpha_{-}(0) \alpha_{-}(1)\right) \backslash\left(P_{n}^{(0)} \cup P_{n}^{(1)}\right): \mathcal{J}^{(0,1)}(p) \neq \emptyset\right\} .
$$

The shift commuting map $\eta_{n}$ is to map a point $p \in P_{n}^{(0,1)}$ to the point $q \in P_{n}$ that is obtained by replacing, in the point $p$, the words $b \in \mathcal{B}(1)$, that appear in $p$ at the indices in $\mathcal{J}_{\circ}^{(0,1)}(p)$, by the word $\Phi_{0}(\Xi(b))$.

A point $p \in P_{n}^{(0,1)}$ can be reconstructed from its image $q$ under $\eta_{n}$ by replacing, in $q$, the word

$$
\beta^{-}(0) h(q) b(q) \in \beta^{-}(0) \mathcal{C}^{*} \mathcal{B}(0,0),
$$

whose prefix $\beta^{-}(0) h(q) \beta^{-}(0)$ is identified as the unique word in $\mathcal{B}(0,0)$ of maximal length that appears openly in $q$, by the word

$$
\beta^{-}(0) h(q) \Xi^{-1}\left(\Phi_{0}^{-1}(b(q))\right) \text {. }
$$

We note that

$$
\left(\nu _ { 0 } \left(\lambda\left(\eta_{n}(p)_{[0, n)}\right), \nu_{1}\left(\lambda\left(\eta_{n}(p)_{[0, n)}\right)\right)=\left(\kappa_{p}+2, \kappa_{p}-1\right), \quad p \in P_{n}^{(0,1)} .\right.\right.
$$

We set

$$
P_{n}^{(1,0)}=P_{n}^{\circ}\left(\alpha^{-}(0) \alpha^{-}(1)\right) \backslash\left(P_{n}^{(0)} \cup P_{n}^{(1)} \cup P_{n}^{(0,1)}\right) .
$$

With the words $b(p) \in \mathcal{B}(1), f(p) \in \mathcal{C}^{*}$, that are given by writing the word in $\mathcal{D}(1,0)$ that appears at the indices in $\mathcal{J}_{0}^{(1,0)}(p)$ as $b(p) f(p) \beta^{-}(0)$, the shift commuting map $\eta_{n}$ is to map a point $p \in P_{n}^{(1,0)}$ to the point $q \in P_{n}(F)$ that is obtained by replacing, in the point $p$, the words in $\mathcal{D}(1,0)$, that appear at the indices in $\mathcal{J}_{\circ}^{(1,0)}(p)$, by the word $\Phi_{0}(\Xi(b(p))) f(p) \beta^{-}(0)$.

A point $p \in P_{n}^{(1,0)}$ can be reconstructed from its image $q$ under $\eta_{n}$ by replacing, in $q$, the word

$$
b(q) \beta^{-}(0) h(q) \beta^{-}(0) \in \mathcal{B}(0,0) \mathcal{C}^{*} \beta^{-}(0),
$$

whose suffix $\left.\beta^{-}(0) h(q) \beta^{-}(0)\right)$ is identified as the unique word of maximal length in $\mathcal{B}(0,0)$ that appears openly in $q$, by the word

$$
\Xi^{-1}\left(\Phi_{0}^{-1}(b(q))\right) h(q) \beta^{-}(0) \text {. }
$$


We note that

$$
\left(\nu _ { 0 } \left(\lambda\left(\eta(p)_{[0, n)}\right), \nu_{1}\left(\lambda\left(\eta(p)_{[0, n)}\right)\right)=\left(\kappa_{p}+2, \kappa_{p}-1\right), \quad p \in \eta_{n}\left(P_{n}^{(1,0)}\right) .\right.\right.
$$

We have produced a partition

$$
P_{n}^{\circ}(\alpha(0) \alpha(0))=P_{n}^{(0)} \cup P_{n}^{(1)} \cup P_{n}^{(0,1)} \cup P_{n}^{(1,0)} .
$$

In points $q \in \eta_{n}\left(P_{n}^{(0,1)}\right)$, the unique word in $\beta^{-}(0) \mathcal{C}^{*} \beta^{-}(0)$ of maximal length that appears openly in $q$ is followed by a word in $\mathcal{C}^{*} \beta^{-}(0)$, whereas, in points $q \in \eta_{n}\left(P_{n}^{(1,0)}\right)$, the unique word in $\beta^{-}(0) \mathcal{C}^{*} \beta^{-}(0)$ of maximal length that appears openly in $q$ is followed by a word in $\mathcal{C}^{*} \beta^{-}$. From this observation and from (P.01[0]), (P.01[1]), (P.01[ $\left.\left.\beta\right]\right)$ and (P.01.1), (P.01.01), (P.01.10) it follows that the images under $\eta_{n}$ of the sets of the partition (P.01) are disjoint. From (P.01[0]), (P.01[1]), (P.01[ $\beta])$ and (P.01.1), (P.01.01), (P.01.10) it follows also that

$$
\eta_{n}\left(P_{n}^{\circ}\left(\alpha_{-}(0) \alpha_{-}(1)\right) \cap P_{n}^{\circ}\left(\alpha_{-}(0)\right)=\emptyset .\right.
$$

We have shown that

$$
\operatorname{card}\left(\mathcal{O}_{n}\left(\alpha_{-}(0) \alpha_{-}(1)\right)\right) \leq \operatorname{card}\left(\bigcup_{\tilde{\mu} \in \mathcal{M} \backslash\{\alpha(0) \alpha(1)\}} \mathcal{O}_{n}\left(\tilde{\mu}^{-}\right)\right) .
$$

Apply Lemma (c).

\section{The remaining multipliers}

Lemma 2. Besides the multipliers $\alpha(0)$ and $\alpha(1)$, the Fibonacci-Dyck shift has no exceptional multipliers.

Proof. Consider a multiplier

$$
\mu \notin\{\alpha(0), \alpha(1), \alpha(0) \alpha(1)\},
$$

of $F$. We construct shift commuting injections

$$
\eta_{n}: P_{n}^{\circ}\left(\mu_{-}\right) \rightarrow \bigcup_{\tilde{\mu} \in \mathcal{M} \backslash\{\mu\}} P_{n}^{\circ}\left(\tilde{\mu}_{-}\right), \quad n>4
$$

We set

$$
P_{n}^{(0,0)}=\left\{p \in P_{n}^{\circ}(\mu): \mathcal{J}^{(0,0)}(p) \neq \emptyset\right\} .
$$

With the word $b(p) \in \mathcal{B}(0,0)$, that appears in $p$ at the indices in $\mathcal{J}_{\circ}^{(0,0)}(p)$, the shift commuting map $\eta_{n}$ is to map a point $p \in P_{n}^{(0,0)}$ to the point $q \in P_{n}$ that is obtained by replacing, in $p$, the words $b(p)$ in $\mathcal{B}(0,0)$, that appear in $p$ at the indices in $\mathcal{J}_{\circ}^{(0,0)}(p)$, by the word $\Phi_{0}^{-1}(b(p))$.

The point $p$ can be reconstructed from its image $q$ under $\eta_{n}$ by replacing, in $q$, the word $c(q)$, that is identified as the unique word in $\mathcal{C}(0)$ of maximal length that appears in $q$, by the word $\Phi_{0}(c(q))$.

We note that

$$
\left(v_{0}\left(\lambda\left(\eta_{n}(p)_{[0, n)}\right), v_{1}\left(\lambda\left(\eta_{n}(p)_{[0, n)}\right)\right)\right)=\left(\kappa_{p} v_{0}(\mu)-2, \kappa_{p} v_{1}(\mu)\right), \quad p \in P_{n}^{(0,0)} .\right.
$$


We set

$$
P_{n}^{(1,1)}=\left\{p \in P_{n}^{\circ}(\mu) \backslash P_{n}^{(0,0)}: \mathcal{J}^{(1,1)}(p) \neq \emptyset\right\} .
$$

With the words

$$
b(p), \in \mathcal{B}(1), \quad f(p) \in \mathcal{C}^{*}, \quad f^{\circ}(p) \in \mathcal{C}^{\circ}(1)^{*},
$$

that are given by writing the word in $\mathcal{D}(1,1)$ that appears in $p$ at the indices in $\mathcal{J}_{\circ}^{(1,1)}(p)$ as

$$
b(p) f(p) \beta^{-} f^{\circ}(p) \beta^{-}(1),
$$

the shift commuting map $\eta_{n}$ is to map a point $p \in P_{n}^{(1,1)}$ to the point $q \in P_{n}(F)$, that is obtained by replacing, in $p$, the words in $\mathcal{D}(1,1)$, that appear in $p$ at the indices in $\mathcal{J}_{\circ}^{(1,1)}(p)$, by the word

$$
\Phi_{0}\left(\Xi(b(p)) f(p) \beta^{+}(0) \Psi\left(f^{\circ}(p)\right) \beta^{-}(0) .\right.
$$

A point $p \in P_{n}^{(1,1)}$ can be reconstructed from its image $q$ under $\eta_{n}$ by replacing, in $q$, the word

$$
\beta^{-}(0) h^{-}(q) \beta^{-}(0) h(q) \beta^{+}(0) h^{+}(q) \beta^{-}(0),
$$

that is identified as the word with the uniquely determined word $\beta^{-}(0) h(q) \beta^{+}(0) \in \mathcal{C}(0)$ of maximal length that appears in $q$ as infix, a uniquely determined openly in $q$ appearing word $h^{+}(q) \beta^{-}(0) \in \mathcal{C}(0)^{*} \beta^{-}(0)$ as suffix, and a uniquely determined word $\beta^{-}(0) h^{-}(q) \in$ $\beta^{-}(0) \mathcal{C}(0)^{*}$ as prefix, by the word

$$
\beta^{-} \Psi^{-1}\left(h^{-}(q)\right) \beta^{-}(1) h(q) \beta^{-} \Psi^{-1}\left(h^{+}(q)\right) \beta^{-}(0) .
$$

We note that

$$
\left(v_{0}\left(\lambda\left(\eta_{n}(p)_{[0, n)}\right)\right), v_{1}\left(\lambda\left(\eta_{n}(p)_{[0, n)}\right)\right)\right)=\left(\kappa_{p} \nu_{0}(\mu)+2, \kappa_{p} v_{1}(\mu)-2\right), p \in P_{n}^{(1,1)} .
$$

We denote by $P_{n}^{(0,1,0)}$ the set of points in

$$
P_{n}^{\circ}(\mu) \backslash\left(P_{n}^{(0,0)} \cup P_{n}^{(1,1)}\right)
$$

such that $\mathcal{J}^{(1)}(p) \neq \varnothing$, and such that the word in $\mathcal{B}(1)$ that appears at the indices in $\mathcal{J}_{\circ}^{(1)}(p)$ is preceded in $p$ by a word in $\beta^{-}(0) \mathcal{C}^{*}$, and followed in $p$ by a word in $\mathcal{C}^{*} \beta^{-}(0)$.

With the word $f(p) \in \mathcal{C}^{*}$, that is given by writing the openly appearing word in $\mathcal{C}^{*} \beta^{-}(0)$, that follows the word $b(p) \in \mathcal{B}(1)$ that appears in $p$ at the indices in $\mathcal{J}_{0}^{(1)}(p)$, as $f(p) \beta^{-}(0)$, the shift commuting map $\eta_{n}$ is to map a point $p \in P_{n}^{(0,1,0)}$ to the point $q \in P_{n}(F)$ that is obtained by replacing, in the point $p$, the words in $\mathcal{B}(1)$, that appear in $p$ at the indices in $\mathcal{J}_{\circ}^{(1)}(p)$ together with the openly appearing words in $\mathcal{C}^{*} \beta^{-}(0)$ that follow them, by the word $\Xi(b(p)) f(p) \beta^{+}(0)$.

Denoting a point $p \in P_{n}^{(0,1,1)}$ by $q^{\prime} \in F$ the point that is obtained from its image $q$ under $\eta_{n}$ by replacing, in $q$, the unique word $c(q) \in \mathcal{C}(0)$ of maximal length, that appears in $q$, by the word $\Phi(c(q))$, one sees that the point $p$ can be reconstructed from $q$ by replacing, in the point $q^{\prime}$, the unique word $c\left(q^{\prime}\right) \in \mathcal{C}(0)$ of maximal length, that appears in $q^{\prime}$, by the word $\Xi^{-1}\left(c\left(q^{\prime}\right)\right)$. 
We note that

$$
\left(v_{0}\left(\lambda\left(\eta_{n}(p)_{[0, n)}\right)\right), v_{1}\left(\lambda\left(\eta_{n}(p)_{[0, n)}\right)\right)\right)=\left(\kappa_{p} v_{0}(\mu)-2, \kappa_{p} v_{1}(\mu)-1\right), p \in P_{n}^{(0,1,0)} .
$$

We denote by $P_{n}^{(\bullet, 1,1)}$ the set of points $p$ in

$$
P_{n}^{\circ}\left(\mu_{-}\right) \backslash\left(P_{n}^{(0,0)} \cup P_{n}^{(0,1,0)}\right)
$$

such that $\mathcal{J}^{(1)}(p) \neq \emptyset$, and such that the words in $\mathcal{B}(1)$ that appear at the indices in $\mathcal{J}_{\circ}^{(1)}(p)$ are followed in $p$ by a word in $\mathcal{C}^{*} \beta^{-} \mathcal{C}^{\circ}(1)^{*} \beta^{-}(1)$.

The shift commuting map $\eta_{n}$ is to map a point $p \in P_{n}^{(\bullet, 1,1)}$ to the point $q \in P_{n}(F)$ that is obtained by replacing, in $p$, the words $b(p) \in \mathcal{B}(1)$, that appear in $p$ at the indices in $\mathcal{J}_{\circ}^{(1)}(p)$, by the word $\Phi_{0}(\Xi(b(p))$.

A point $p \in P_{n}^{(\bullet, 1,1)}$ can be reconstructed from its image $q$ under $\eta_{n}$ by replacing, in $q$, the word $h(q)$, that is identified as the unique word in $\mathcal{B}(0,0)$ of maximal length that appears in $q$, by the word $\Xi^{-1}\left(\Phi_{0}^{-1}(h(q))\right)$.

We note that

$$
\left(v _ { 0 } \left(\lambda\left(\eta_{n}(p)_{[0, n)}\right), v_{1}\left(\lambda\left(\eta_{n}(p)_{[0, n)}\right)=\left(\kappa_{p} v_{0}(\mu)+2, \kappa_{p} v_{1}(\mu)-1\right), p \in P_{n}^{(\bullet, 1,1)} \cdot(\bullet .1 .1)\right.\right.\right.
$$

We denote by $P_{n}^{(1,1, \bullet)}$ the set of points in

$$
P_{n}^{\circ}\left(\mu_{-}\right) \backslash\left(P_{n}^{(0,0)} \cup P_{n}^{(0,1,0)} \cup P_{n}^{(\bullet, 1,1)}\right)
$$

such that $\mathcal{J}^{(1)}(p) \neq \varnothing$, and such that the word in $\mathcal{B}(1)$ that appears at indices in $\mathcal{J}_{\circ}^{(1)}(p)$ is preceded in $p$ by a word in $\beta^{-} \mathcal{C}^{\circ}(1)^{*} \beta^{-}(1) \mathcal{C}^{*}$.

The shift commuting map $\eta_{n}$ is to map a point $p \in P_{n}^{(1,1, \bullet)}$ to the point $q \in P_{n}(F)$ that is obtained by replacing, in $p$, the words $b(p) \in \mathcal{B}(1)$, that appear in $p$ at the indices in $\mathcal{J}_{\circ}^{(1)}(p)$, by the word $\Xi(b(p))$.

A point $p \in P_{n}^{(1,1, \bullet)}$ can be reconstructed from its image $q$ under $\eta_{n}$ by replacing, in $q$, the word $c(q)$, that is identified as the unique word in $\mathcal{C}(0)$ of maximal length that appears in $q$, by the word $\Xi^{-1}(c(q))$.

We note that

$$
\left(v _ { 0 } \left(\lambda\left(\eta_{n}(p)_{[0, n)}\right), v_{1}\left(\lambda\left(\eta_{n}(p)_{[0, n)}\right)=\left(\kappa_{p} v_{0}(\mu), \kappa_{p} v_{1}(\mu)-1\right), \quad p \in P_{n}^{(1,1, \bullet)} .\right.\right.\right.
$$

We set

$$
\left.P_{n}^{(0,1)}=\left\{p \in P_{n}^{\circ}\left(\mu_{-}\right) \backslash\left(P_{n}^{(0,0)} \cup P_{n}^{(0,1,0)} \cup P_{n}^{(\bullet, 1,1)}\right) \cup P^{(1,1, \bullet)}\right): \mathcal{J}^{(1,0)}(p) \neq \emptyset\right\} .
$$

The shift commuting map $\eta_{n}$ is to map a point $p \in P_{n}^{(0,1)}$ to the point $q \in P_{n}(F)$ that is obtained by replacing, in $p$, the words $b(p) \in \mathcal{B}(1)$, that appear at the indices in $\mathcal{J}_{\circ}^{(0,1)}(p)$, by the word $\Phi_{0}(\Xi(b(p)))$.

The point $p \in P_{n}^{(0,1)}$ can be reconstructed from its image $q$ under $\eta_{n}$ by replacing, in $q$, the word $\beta^{-}(0) g(q) \beta^{-}(0)$, that is identified as the unique word in $\mathcal{B}(0,0)$ of maximal length that appears in $q$, by the word $\beta^{-}(0) g(q) \beta^{-}$, and the open appearances $h(q) \beta^{-}(0)$ of a word in $\mathcal{C}(0)^{*} \beta^{-}(0)$, that follow the word $\beta^{-}(0) g(q) \beta^{-}(0)$ in $q$, by the word $\Psi^{-1}(h(q)) \beta^{-}(1)$. 
We note that

$\left(\nu_{0}\left(\lambda\left(\eta(p)_{[0, n)}\right)\right), \nu_{1}\left(\lambda\left(\eta(p)_{[0, n)}\right)\right)\right)=\left(\kappa_{p} \nu_{0}(\mu)+2, \kappa_{p} v_{1}(\mu)-1\right), \quad p \in P_{n}^{(0,1)}$.

We set

$$
P_{n}^{(1,0)}=P_{n}^{\circ}\left(\mu_{-}\right) \backslash\left(P_{n}^{(0,0)} \cup P_{n}^{(0,1,0)} \cup P_{n}^{(\bullet, 1,1)} \cup P^{(1,1, \bullet)} \cup P_{n}^{(0,1)}\right) .
$$

With the words

$$
b(p) \in \mathcal{B}, \quad f(p) \in \mathcal{C}^{*},
$$

that are given by writing the word in $\mathcal{D}(1,0)$ that appears at the indices in $\mathcal{J}_{0}^{(1,0)}(p)$ as

$$
b(p) f(p) \beta^{-}(0),
$$

the shift commuting map $\eta_{n}$ is to map a point $p \in P_{n}^{(1,0)}$ to the point $q \in P_{n}(F)$, that is obtained by replacing, in $p$, the words $b(p) f(p) \beta^{-}(0)$, that appear at the indices in $\mathcal{J}_{\circ}^{(1,0)}(p)$, by the word

$$
\Phi_{0}\left(\Xi(b(p)) f(p) \beta^{+}(0) .\right.
$$

The point $p \in P_{n}^{(1,0)}$ can be reconstructed from its image $q$ under $\eta_{n}$ by replacing, in $q$, the word $\beta^{-}(0) g(q) \beta^{+}(0)$, that is identified as the unique word in $\mathcal{C}(0)$ of maximal length that appears in $q$, by the word $\beta^{-}(1) g(q) \beta^{-}(0)$, and the word $\beta^{-}(0) h(q) \in \beta^{-}(0) \mathcal{C}^{*}$, that precedes the word $\beta^{-}(0) g(q) \beta^{+}(0)$ in $q$, by the word $\beta^{-} \Psi^{-1}(h(q))$.

We note that

$$
\left(v_{0}\left(\lambda\left(\eta(p)_{[0, n)}\right)\right), v_{1}\left(\lambda\left(\eta(p)_{[0, n)}\right)\right)\right)=\left(\kappa_{p} v_{0}(\mu), \kappa_{p} v_{1}(\mu)-1\right), \quad p \in P_{n}^{(1,0)} .
$$

We have produced a partition

$$
P_{n}^{\circ}\left(\mu_{-}\right)=P_{n}^{(0,0)} \cup P_{n}^{(0,1,0)} \cup P_{n}^{(\bullet, 1,1)} \cup P_{n}^{(1,1, \bullet)} \cup P_{n}^{(0,1)} \cup P_{n}^{(1,0)} .
$$

In a point $q \in \eta_{n}\left(P_{n}^{(\bullet, 1,1)}\right)$, the word in $\mathcal{B}(0,0)$ of maximal length that appears in $q$ is followed by an open appearance of a word in $\mathcal{C}^{*} \mathcal{B}(1)$, whereas, in the points $q \in \eta_{n}\left(P_{n}^{(0,1)}\right)$, this word is followed by an open appearance of a word in $\mathcal{C}^{*} \beta^{-}(0)$. Also, in a point $q \in \eta_{n}\left(P_{n}^{(1,1, \bullet)}\right)$, the word in $\mathcal{C}(0)$ of maximal length that appears in $q$ is preceded by a word in $\mathcal{B}(1) \mathcal{C}^{*}$, whereas, in a point $q \in \eta_{n}\left(P_{n}^{(1,0)}\right)$, this word is preceded by a word in $\beta^{-}(0) \mathcal{C}^{*}$.

It follows from these observations and from $(00),(0,1,0),(\bullet, 1,1),(1,1, \bullet),(0,1)$ and $(1,0)$ that the images under $\eta_{n}$ of the elements of the partition $(\mathrm{P})$ are disjoint. From $(0,0)$, $(0,1,0),(\bullet, 1,1),(1,1, \bullet),(0,1)$ and $(1,0)$ it also follows that

$$
\eta_{n}\left(P_{n}^{\circ}\left(\mu_{-}\right)\right) \cap P_{n}^{\circ}\left(\mu_{-}\right)=\emptyset .
$$

We have shown that

$$
\operatorname{card} \mathcal{O}_{n}\left(\mu_{-}\right) \leq \operatorname{card}\left(\bigcup_{\tilde{\mu} \in \mathcal{M} \backslash\left\{\mu^{-}\right\}} \mathcal{O}_{n}\left(\tilde{\mu}_{-}\right)\right) .
$$

The lemma follows now from Lemma (c) and Lemma 1. 


\section{An embedding theorem}

Let $P_{k}(\alpha(0))$ denote the set of points of $F$ of period $k$ with multiplier $\alpha(0)$, let $P_{k}(\alpha(1))$ denote the set of points of $F$, of period $k$, with multiplier $\alpha(1)$ and let $P_{k}(\mathbf{1})$ denote the set of points of $F$, of period $k$, that are neutral. Denote by $\zeta_{1}$ the zeta function of the neutral periodic points of $F$, by $\zeta_{\alpha(0)}$ the zeta function of the periodic points of $F$ with multiplier $\alpha(0)$ and by $\zeta_{\alpha(1)}$ the zeta function of the periodic points of $F$ with multiplier $\alpha(1)$.

LEMMA 3.

$$
\begin{aligned}
& \liminf _{k \rightarrow \infty} \frac{1}{k} \log \operatorname{card}\left(P_{k}(\mathbf{1}) \cup P_{k}(\alpha(0))\right) \\
& \quad=\liminf _{k \rightarrow \infty} \frac{1}{k} \log \operatorname{card}\left(P_{k}(\mathbf{1}) \cup P_{k}(\alpha(1))\right)=\frac{3}{2} \log 3-\log 2 .
\end{aligned}
$$

Proof. Set

$$
\xi(z)=\frac{2}{\sqrt{3}} \sin \left(\frac{1}{3} \arcsin \frac{3 \sqrt{3}}{2} z\right), \quad 0 \leq z \leq \frac{2}{3 \sqrt{3}} .
$$

By [KM, (4.8)] we have the generating functions

$$
g_{C^{\circ}(1)^{\star}}(z)=\frac{\xi(z)}{z}
$$

and

$$
g_{C^{\star}}(z)=\frac{\xi(z)^{2}}{z^{2}},
$$

and it follows that

$$
\zeta_{\mathbf{1}}(z)=g_{\mathcal{C}^{\circ}(1)^{\star}}(z) g_{\mathcal{C}^{\star}}(z)=\frac{\xi(z)^{3}}{z^{3}} .
$$

Using the circular code $\mathcal{C}^{\star} \beta^{-}(0)$ one finds from (5.2) that

$$
\zeta_{\alpha(0)}(z)=\left(\frac{z}{z-\xi(z)^{2}}\right)^{2}
$$

and using the circular code $\beta^{-}(1) \mathcal{C}^{\star} \beta^{-} \mathcal{C}^{\circ}(1)^{\star}$ one finds, from (5.1) and (5.2), that

$$
\zeta_{\alpha(1)}(z)=\left(\frac{z}{z-\xi(z)^{3}}\right)^{2} .
$$

(See, for example, [P, §5] or [KM, §2].) The lemma follows from (5.4), (5.5) and (5.3).

Let $P_{k}^{+}$denote the set of points of $F$ of period $k$ with positive multiplier, where $k \in \mathbb{N}$. Let $\mathcal{O}_{k}^{+}$denote the set of orbits of $F$ of length $k$ with positive multiplier, let $\mathcal{O}_{k}(\alpha(0))$ denote the set of orbits of $F$ of length $k$ with multiplier $\alpha(0)$, let $\mathcal{O}_{k}(\alpha(1))$ denote the set of orbits of $F$ of length $k$ with multiplier $\alpha(1)$ and let $\mathcal{O}_{k}(\mathbf{1})$ denote the set of orbits of $F$ of length $k$ that are neutral, where $k \in \mathbb{N}$.

THEOREM. Let $Y$ be an irreducible subshift of finite type. Let $\mathcal{O}_{k}(Y)$ be its set of periodic orbits of length $k \in \mathbb{N}$, and let $h_{Y}$ be its entropy. An embedding of $Y$ into the FibonacciDyck shift exists if and only if at least one of the following conditions is satisfied. 
(a)

$$
\operatorname{card}\left(\mathcal{O}_{k}(Y)\right) \leq \operatorname{card}\left(\mathcal{O}_{k}(\mathbf{1}) \cup \mathcal{O}_{k}(\alpha(0))\right), \quad k \in \mathbb{N},
$$

and

$$
h(Y)<\frac{3}{2} \log 3-\log 2 .
$$

(b)

$$
\operatorname{card}\left(\mathcal{O}_{k}(Y)\right) \leq \operatorname{card}\left(\mathcal{O}_{k}(\mathbf{1}) \cup \mathcal{O}_{k}(\alpha(1))\right), \quad k \in \mathbb{N},
$$

and

$$
h(Y)<\frac{3}{2} \log 3-\log 2 .
$$

(c)

$$
\operatorname{card}\left(\mathcal{O}_{k}(Y)\right) \leq \operatorname{card}\left(\mathcal{O}_{k}(\mathbf{1}) \cup \mathcal{O}_{k}^{+}\right), \quad k \in \mathbb{N},
$$

and

$$
h(Y)<3 \log 2-\log 3 .
$$

Proof. The theorem results from an application of [HIK, Theorem 5.8] that uses Lemma (a) and Lemmas 1-3 and that takes into account that only the exceptional multipliers (in this case the multipliers $\alpha(0)$ and $\alpha(1)$ ) contribute to the possibility of an embedding beyond the case of negative (or positive) multipliers. In [HIK, Theorem 5.8], the entropy condition in (a) reads

$$
h(Y)<\liminf _{k \rightarrow \infty} \frac{1}{k} \log \operatorname{card}\left(P_{k}(\mathbf{1}) \cup P_{k}(\alpha(0))\right),
$$

the entropy condition in (b) reads

$$
h(Y)<\liminf _{k \rightarrow \infty} \frac{1}{k} \log \operatorname{card}\left(P_{k}(\mathbf{1}) \cup P_{k}(\alpha(1))\right)
$$

and the entropy condition in (c) reads

$$
h(Y)<\liminf _{k \rightarrow \infty} \frac{1}{k} \log \operatorname{card}\left(P_{k}(\mathbf{1}) \cup P_{k}^{+}\right) .
$$

For (a) and (b) apply Lemma 3. For (c) note that, by Lemma (a), the right-hand side of the inequality is equal to the topological entropy of $F$, which is known to be $3 \log 2-$ $\log 3$ [KM, §4].

By Lemma (a),

$$
\begin{aligned}
\operatorname{card}\left(\mathcal{O}_{k}^{+}\right) & =\frac{1}{2} \operatorname{card}\left(\mathcal{O}_{k} \backslash \mathcal{O}_{k}(\mathbf{1})\right), \\
\operatorname{card}\left(\mathcal{O}_{k}(\alpha(0))\right) & =2 \operatorname{card}\left(\mathcal{O}_{k}\left(\alpha^{-}(0)\right),\right. \\
\operatorname{card}\left(\mathcal{O}_{k}(\alpha(1))\right) & =2 \operatorname{card}\left(\mathcal{O}_{k}\left(\alpha^{-}(1)\right), \quad k \in \mathbb{N} .\right.
\end{aligned}
$$

Denote the set of points of $F$ of period $k$ by $P_{k}, k \in \mathbb{N}$, and denote by $\zeta$ the zeta function of $F$. The sequence $\left(\operatorname{card}\left(\mathcal{O}_{k}\right)\right)_{k \in \mathbb{N}}$ can be obtained by Möbius inversion from the sequence $\left(\operatorname{card}\left(P_{k}\right)_{k \in \mathbb{N}}\right.$ that enters into the zeta function $\zeta(z)=e^{\sum_{n \in \mathbb{N}}\left(\left(\operatorname{card}\left(P_{n}\right) z^{n}\right) / n\right)}$ of $F$. The same applies to the sequences

$$
\operatorname{card}\left(\mathcal{O}_{k}(\mathbf{1})\right)_{k \in \mathbb{N}}, \quad \operatorname{card}\left(P_{k}(\alpha(0))\right)_{k \in \mathbb{N}}, \quad \operatorname{card}\left(\mathcal{O}_{k}(\alpha(1))\right)_{k \in \mathbb{N}} .
$$


Therefore, by (5.6), the information that is relevant for each of the conditions (a), (b) and (c) of Theorem (5.1) is (in principle) contained in the zeta functions $\zeta, \zeta_{\mathbf{1}}$, and $\zeta_{\alpha(0)}, \zeta_{\alpha(1)}$. $\zeta$ is also known. From [KM, (4.12)],

$$
\zeta(z)=\frac{\xi(z)}{z\left(2 \xi(z)^{2}+\xi(z)-1\right)^{2}} .
$$

We note that the zeta function $\zeta_{+}(z)$ of the periodic point with positive multiplier is also known: by Lemma (a),

$$
\zeta_{+}(z)=\sqrt{\zeta_{\mathbf{1}}(z)^{-1} \zeta(z)}=\frac{\xi(z)^{2}}{z^{2}\left(2 \xi(z)^{2}+\xi(z)-1\right)} .
$$

\section{The multiplier $\alpha(0)$}

PROPOSITION M0. The multiplier $\alpha(0)$ is exceptional only at periods one and three.

Proof. By Lemma (b) the multiplier $\alpha(0)$ is is not exceptional at periods two, four, five and six. Let

$$
n \geq 7 \text {. }
$$

We construct a shift commuting injection

$$
\eta_{n}: P_{n}^{\circ}\left(\alpha_{-}(0)\right) \rightarrow \bigcup_{\tilde{\mu} \in \mathcal{M} \backslash\{\alpha(0)\}} P_{n}^{\circ}\left(\tilde{\mu}_{-}\right) .
$$

Set

$$
I(i)=\max \left\{i^{(0)} \in \mathcal{I}^{(0)}: i^{(0)}<i\right\}, \quad i \in \mathcal{I}^{(0)}(p), \quad p \in P_{n}^{\circ}\left(\alpha^{-}(0)\right) .
$$

Let $P_{n}^{(1)}$ be the set of points $p \in P_{n}^{\circ}\left(\alpha^{-}(0)\right)$ such that the set $\mathcal{I}^{\langle 1\rangle}$ of indices $i^{0} \in \mathcal{I}^{(0)}$ and such that $p_{(I(i), i)} \in \mathcal{Q}_{1}$ is not empty.

For $p \in P_{n}^{(1)}$, we denote by $\mathcal{I}_{\circ}^{\langle 1\rangle}(p)$ the set of indices $i_{\circ}^{(1)} \in \mathcal{I}^{\langle 1\rangle}(p)$ such that the word $p_{\left(I\left(i_{\circ}^{(1)}\right), i_{\circ}^{(1)}\right]}$ is lexicographically the smallest among the words

$$
p_{\left(I\left(i^{(1)}\right), i^{(1)}\right]}, \quad i^{(1)} \in \mathcal{I}^{\langle 1\rangle}(p) .
$$

With the word $f(p) \in \mathcal{Q}_{1}$ that is given by writing

$$
p_{\left(I\left(i_{\circ}^{(1)}\right), i_{\circ}^{(1)}\right]}=f(p) \beta_{-}(0), \quad i_{\circ}^{(1)} \in \mathcal{I}_{\circ}^{\langle 1\rangle}(p),
$$

the shift commuting map $\eta_{n}$ is to map a point $p \in P_{n}^{(1)}$ to the point $p \in P_{n}^{\circ}(F)$ that is obtained by replacing, in the point $p$, the words $f(p) \beta_{-}(0)$, that appear at the indices in $\mathcal{I}_{\circ}^{\langle 1\rangle}(p)$, by $\Delta_{1}(f) \beta_{-}(0)$.

For $q \in \eta_{n}\left(P_{n}^{(1)}\right)$, there is a unique word $b(q) \in \mathcal{B}(1,1)$ that appears openly in $q$, and a point $p \in P_{n}^{(1)}$ that can be reconstructed from its image $q$ under $\eta_{n}$ by replacing, in $q$, the word $b(q)$, when it appears openly in $q$, by the word $\Phi_{1}^{-1}(b(q))$.

We note that

$$
\left(v_{0}\left(\lambda\left(\eta(p)_{[0, n)}\right)\right), v_{1}\left(\lambda\left(\eta(p)_{[0, n)}\right)\right)\right)=\left(\kappa_{p}, 2\right), \quad p \in P_{n}^{(1)} .
$$


Let $P_{n}^{(\beta)}$ be the set of points

$$
\left.p \in P_{n}^{\circ}\left(\alpha^{-}(0)\right) \backslash P_{n}^{(1)}\right)
$$

such that the set $\mathcal{I}^{\langle\beta\rangle}(p)$ of indices $i^{(\beta)} \in \mathcal{I}^{(0)}(p)$, at which there appears a word in $\beta^{-} \beta^{+}\left(\mathcal{C}(0)^{*} \backslash\{\epsilon\}\right) \beta^{-}(0)$, is not empty.

For $p \in P_{n}^{(\beta)}$, we denote by $\mathcal{I}_{\circ}^{\langle\beta\rangle}(p)$ the set of indices $i_{\circ}^{(\beta)} \in \mathcal{I}^{\langle\beta\rangle}(p)$ such that the word $p_{\left(i_{\circ}^{(\beta)}-n, i_{\circ}^{(\beta)}\right]}$ is lexicographically the smallest among the words

$$
\left.p_{(i(\beta)}-n, i^{(\beta)}\right], \quad i^{(\beta)} \in \mathcal{I}^{\langle\beta\rangle}(p) .
$$

With the word $f(p) \in \mathcal{C}(0)^{*}$ that is given by writing

$$
p_{\left(I\left(i_{\circ}^{(\beta)}\right), i_{\circ}^{(\beta)}\right]}=f(p), \quad i_{\circ}^{(\beta)} \in \mathcal{I}_{\circ}^{\langle\beta\rangle}(p),
$$

the shift commuting map $\eta_{n}$ is to map a point $p \in P_{n}^{(\beta)}$ to the point $p \in P_{n}^{\circ}(F)$ that is obtained by replacing, in the point $p$, the word $\beta^{-} \beta^{+} f(p)$, that appears in $p$ at the indices in $\mathcal{I}_{\circ}^{\langle\beta\rangle}(p)-1$, by the word $\beta^{-} \Psi(f(p)) \beta^{-}(1)$.

For a point $q \in \eta_{n}\left(P_{n}^{(\beta)}\right)$ there is a unique word $b(q) \in \mathcal{B}(1)$ that appears openly in $q$, and a point $p \in P_{n}^{(\beta)}$ that can be reconstructed from its image $q$ under $\eta_{n}$ by replacing, in $q$, the word $b(q)$, when it appears openly in $q$, by the word $\beta^{-} \beta^{+} \Psi^{-1}(b(q))$.

We note that

$$
\left(v_{0}\left(\lambda\left(\eta(p)_{[0, n)}\right)\right), v_{1}\left(\lambda\left(\eta(p)_{[0, n)}\right)\right)\right)=\left(\kappa_{p}, 2\right), \quad p \in P_{n}^{(\beta)} .
$$

Let $P_{n}^{(\beta, 0)}$ be the set of points

$$
p \in P_{n}^{\circ}\left(\alpha^{-}(0)\right) \backslash\left(P_{n}^{(1)} \cup P_{n}^{(\beta)}\right)
$$

such that the set $\mathcal{I}^{\langle\beta, 0\rangle}(p)$ of indices $i^{(\beta, 0)} \in \mathcal{I}^{(0)}(p)$, at which there appears openly the word $\beta^{-} \beta^{+} \beta^{-}(0)$, is not empty.

For $p \in P_{n}^{(\beta, 0)}$, we denote by $\mathcal{I}_{\circ}^{\langle\beta, 0\rangle}(p)$ the set of indices $i_{\circ}^{(\beta, 0)} \in \mathcal{I}^{\langle\beta, 0\rangle}(p)$ such that the word $p_{\left(i_{\circ}^{(\beta, 0)}-n,\left(i_{\circ}^{(\beta, 0)}\right]\right.}$ is lexicographically the smallest among the words

$$
p_{\left(i^{(\beta, 0)}-n, i^{(\beta, 0)}\right]}, \quad i^{(\beta, 0)} \in \mathcal{I}^{\langle\beta, 0\rangle}(p) .
$$

The shift commuting map $\eta_{n}$ is to map a point $p \in P_{n}^{(\beta, 0)}$ to the point $p \in P_{n}^{\circ}(F)$ that is obtained by replacing, in the point $p$, the word $\beta^{-} \beta^{+}$, that appears in $p$ at the indices in $\mathcal{I}_{\circ}^{\langle\beta, 0\rangle}(p)-1$, by the word $\beta^{-} \beta^{-}(1)$.

In a point of $\eta_{n}\left(P_{n}^{(\beta, 0)}\right)$ the word $\beta^{-} \beta^{-}(1)$ appears openly, and a point $p \in P_{n}^{(\beta, 0)}$ can be reconstructed from its image $q$ under $\eta_{n}$ by replacing, in $q$, the word $\beta^{-} \beta^{-}(1)$, when it appears openly in $q$, by the word $\beta^{-} \beta^{+}$.

We note that

$$
\left(\nu_{0}\left(\lambda\left(\eta(p)_{[0, n)}\right)\right), v_{1}\left(\lambda\left(\eta(p)_{[0, n)}\right)\right)\right)=\left(\kappa_{p}, 2\right), \quad p \in P_{n}^{(\beta, 0)} .
$$

Let $P_{n}^{(0,2)}$ be the set of points

$$
p \in P_{n}^{\circ}\left(\alpha^{-}(0)\right) \backslash\left(P^{(1)} \cup P_{n}^{(\beta)} \cup P_{n}^{(\beta, 0)}\right)
$$


such that

$$
v_{0}(p) \geq 2 .
$$

For $p \in P_{n}^{(0,2)}$, we denote by $\mathcal{I}_{\circ}^{\langle 0,2\rangle}(p)$ the set of indices $i \in \mathcal{I}^{(0)}(p)$ such that

$$
i-I(i)>1 \text {. }
$$

For $p \in P_{n}^{(0,2)}$, we denote by $\mathcal{I}_{\circ}^{\langle 0,2\rangle}(p)$ the set of indices $i_{\circ}^{(0,2)} \in \mathcal{I}^{\langle 0,2\rangle}(p)$ such that the word $p_{\left(i_{\circ}^{(0,2)}-n, i_{\circ}^{(0,2)}\right]}$ is lexicographically the smallest among the words

$$
p_{\left(i(0,2)-n, i^{(0,2)}\right]}, \quad i^{(0,2)} \in \mathcal{I}^{\langle 0,2\rangle}(p) .
$$

With the word $f(p) \in \mathcal{C}(0)^{*}$, that is given by writing

$$
p_{\left[I\left(i_{\circ}^{\langle 0,2\rangle}\right), i_{\circ}^{\langle 0,2\rangle}\right]}=\beta^{-}(0) f(p) \beta^{-}(0), \quad i^{(0,2)} \in \mathcal{I}_{\circ}^{\langle 0,2\rangle}(p),
$$

the shift commuting map $\eta_{n}$ is to map a point $p \in P_{n}^{(0,2)}$ to the point $p \in P_{n}^{\circ}(F)$ that is obtained by replacing, in the point $p$, the words $\beta^{-}(0) f(p) \beta^{-}(0)$, that appear in $p$ at the indices in $\mathcal{I}_{\circ}^{\langle 0,2\rangle}(p)$, by the word $\beta^{-} \Psi^{-1}(f(p)) \beta^{-}(1)$.

For a point $q \in \eta_{n}\left(P_{n}^{(0,2)}\right)$ there is a unique word $b(q) \in \mathcal{B}(1)$ that appears openly in $q$, and a point $p \in P_{n}^{(0,2)}$ that can be reconstructed from its image $q$ under $\eta_{n}$ by replacing, in $q$, the word $b(q)$, when it appears openly in $q$, by the word $\beta^{-} \Psi^{-1}(b(q)) \beta^{-}$.

We note that

$$
\left(\nu_{0}\left(\lambda\left(\eta(p)_{[0, n)}\right)\right), \nu_{1}\left(\lambda\left(\eta(p)_{[0, n)}\right)\right)\right)=\left(\kappa_{p}-2,1\right), \quad p \in P_{n}^{(0,2)} .
$$

Let $P_{n}^{(0,1, l)}$ be the set of points

$$
p \in P_{n}^{\circ}\left(\alpha^{-}(0)\right) \backslash\left(P^{(1)} \cup P_{n}^{(\beta)} \cup P_{n}^{(\beta, 0)} \cup P_{n}^{(0,2)}\right)
$$

such that

$$
p_{(i-n, i]} \in \beta^{-}(0) \beta^{+}(0) \mathcal{C}(0)^{*} \beta^{-}(0), \quad i \in \mathcal{I}^{(0)}(p) .
$$

With the word $f(p) \in \beta^{-}(0) \beta^{+}(0) \mathcal{C}(0)^{*} \beta^{-}(0)$, that is given by writing

$$
p_{(i-n, i]}=\beta^{-}(0) \beta^{+}(0) f(p) \beta^{-}(0), \quad i \in \mathcal{I}^{(0)}(p),
$$

the shift commuting map $\eta_{n}$ is to map a point $p \in P_{n}^{(0,1, l)}$ to the point $q \in P_{n}^{\circ}(F)$ that is given by

$$
q_{(i-n, i]}=\beta^{-} \beta^{-}(1) f(p) \beta^{-}(0), \quad i \in \mathcal{I}^{(0)}(p) .
$$

For a point $q \in \eta_{n}\left(P_{n}^{(0,1, l)}\right)$,

$$
q_{(i-n, i]} \in \beta^{-} \beta^{-}(1) \mathcal{C}(0)^{*} \beta^{-}(0), \quad i \in \mathcal{I}^{(0)}(q),
$$

and with the word $a(q) \in \mathcal{C}(0)^{*}$, that is given by writing

$$
q_{(i-n, i]}=\beta^{-} \beta^{-}(1) a(q) \beta^{-}(0), \quad i \in \mathcal{I}^{(0)}(q),
$$

a point $p \in P_{n}^{(0,1, l)}$ can be reconstructed from its image $q$ under $\eta_{n}$ as the point in $P_{n}^{\circ}(F)$ that is given by

$$
\left.p_{(i-n, i]}=\beta^{-}(0) \beta^{+}(0) a(q)\right) \beta^{-}(0), \quad i \in \mathcal{I}^{(0)}(q) .
$$


We note that

$$
\left(v_{0}\left(\lambda\left(\eta(p)_{[0, n)}\right)\right), v_{1}\left(\lambda\left(\eta(p)_{[0, n)}\right)\right)\right)=(1,1), \quad p \in P_{n}^{(0,1, l)} .
$$

Let $P_{n}^{(0,1, m)}$ be the set of points

$$
p \in P_{n}^{\circ}\left(\alpha^{-}(0)\right) \backslash\left(P_{n}^{(1)} \cup P_{n}^{(\beta)} \cup P_{n}^{(\beta, 0)} \cup P_{n}^{(0,2)}\right)
$$

such that

$$
p_{(i-n, i]} \in \beta^{-}(0)\left(\mathcal{C}(0)^{*} \backslash\{\epsilon\}\right) \beta^{+}(0)\left(\mathcal{C}(0)^{*} \backslash\{\epsilon\}\right) \beta^{-}(0), \quad i \in \mathcal{I}^{(0)}(p) .
$$

With the words $f(p) \in \mathcal{C}(0)^{*}, g(p) \in \mathcal{C}(0)^{*}$, that are given by writing

$$
p_{(i-n, i]}=\beta^{-}(0) f(p) \beta^{+}(0) g(p) \beta^{-}(0), \quad i \in \mathcal{I}^{(0)}(p),
$$

the shift commuting map $\eta_{n}$ is to map a point $p \in P_{n}^{(0,1, m)}$ to the point $q \in P_{n}^{\circ}(F)$ that is given by

$$
q_{(i-n, i]}=\beta^{-}(1) f(p) \beta^{-}(0) g(p) \beta^{-}, \quad i \in \mathcal{I}^{(0)}(p) .
$$

For a point $q \in \eta_{n}\left(P_{n}^{(0,1 . m)}\right)$,

$$
q_{(i-n, i]} \in \mathcal{C}(0)^{*} \beta^{-}(0) \mathcal{C}(0)^{*} \beta^{-} \beta^{-}(1), \quad i \in \mathcal{I}^{(1)}(q),
$$

and with the words $a(q) \in \mathcal{C}(0)^{*}, b(q) \in \mathcal{C}^{*}$, that are given by writing

$$
q_{[i, i+n}=a(q) \beta^{-}(0) b(q) \beta^{-} \beta^{-}(1) . \quad i \in \mathcal{I}^{(1)}(q),
$$

a point $p \in P_{n}^{(0,1,0)}$ can be reconstructed from its image $q$ under $\eta_{n}$ as the point that is given by

$$
p_{(i-n, i]}=a(q) \beta^{+}(0) b(q) \beta^{-}(0) \beta^{-}(0), \quad i \in \mathcal{I}^{(1)}(q) .
$$

We note that

$$
\left(v_{0}\left(\lambda\left(\eta(p)_{[0, n)}\right)\right), v_{1}\left(\lambda\left(\eta(p)_{[0, n)}\right)\right)\right)=(1,1), \quad p \in P_{n}^{(0,1,0)} .
$$

Let $P_{n}^{(0,1, r)}$ be the set of points

$$
p \in P_{n}^{\circ}\left(\alpha^{-}(0)\right) \backslash\left(P_{n}^{(1)} \cup P_{n}^{(\beta)} \cup P_{n}^{(\beta, 0)} \cup P_{n}^{(0,2)}\right)
$$

such that

$$
p_{(i-n, i]} \in \beta^{-}(0) \beta^{-}(0)\left(\mathcal{C}(0)^{*} \backslash\{\epsilon\}\right) \beta^{+}(0) \mathcal{C}(0)^{*} \beta^{+}(0) \beta^{-}(0), \quad i \in \mathcal{I}^{(0)}(p) .
$$

With the words $f(p) \in \mathcal{C}(0)^{*}, g(p) \in \mathcal{C}(0)^{*}$, that are given by writing

$$
p_{(i-n, i]}=\beta^{-}(0) f(p) \beta^{+}(0) g(p) \beta^{-}(0), \quad i \in \mathcal{I}^{(0)}(p),
$$

the shift commuting map $\eta_{n}$ is to map a point $p \in P_{n}^{(0,1, r)}$ to the point $q \in P_{n}^{\circ}(F)$ that is given by

$$
q_{(i-n, i]}=\beta^{-} \beta^{-}(1) f(p) \beta^{-}(0) g(p) \beta^{-}(0) \beta^{-}(0), \quad i \in \mathcal{I}^{(0)}(p) .
$$

For a point $q \in \eta_{n}\left(P_{n}^{(0,1, r)}\right)$,

$$
q_{(i-n, i]} \in \mathcal{C}(0)^{*} \beta^{-}(0) \mathcal{C}(0)^{*} \beta^{-}(0) \beta^{-}(0) \beta^{-} \beta^{-}(1), \quad i \in \mathcal{I}^{(1)}(q),
$$


and with the words $a(q) \in \mathcal{C}(0)^{*}, b(q) \in \mathcal{C}(0)^{*}$, that are given by writing

$$
q_{[i, i+n}=a(q) \beta^{-}(0) b(q) \beta^{-}(0) \beta^{-}(0) \beta^{-} \beta^{-}(1) . \quad i \in \mathcal{I}^{(1)}(q),
$$

a point $p \in P_{n}^{(0,1, r)}$ can be reconstructed from its image $q$ under $\eta_{n}$ as the point that is given by

$$
p_{(i-n, i]}=a(q) \beta^{-}(0) b(q) \beta^{-}(0) \beta^{-} \beta^{-}(1), \quad i \in \mathcal{I}^{(1)}(q) .
$$

We note that

$$
\left(\nu_{0}\left(\lambda\left(\eta(p)_{[0, n)}\right)\right), v_{1}\left(\lambda\left(\eta(p)_{[0, n)}\right)\right)\right)=(3,1), \quad p \in P_{n}^{(0,1, r)} .
$$

Let $P_{n}^{(0,1, r, \beta)}$ be the set of points

$$
p \in P_{n}^{\circ}\left(\alpha^{-}(0)\right) \backslash\left(P^{(1)} \cup P_{n}^{(\beta)} \cup P_{n}^{(\beta, 0)} \cup P_{n}^{(0,2)}\right)
$$

such that

$$
p_{(i-n, i]} \in \beta^{-}(0) \beta^{-}(0) \beta^{+}(0)\left(\mathcal{C}(0)^{*} \backslash \mathcal{C}(0)^{*}\right) \beta^{+}(0) \beta^{-}(0), \quad i \in \mathcal{I}^{(0)}(p) .
$$

With the word $f(p) \in \mathcal{C}(0)^{*}$, that is given by writing

$$
p_{(i-n, i]}=\beta^{-}(0) \beta^{-}(0) \beta^{+}(0) f(p) \beta^{+}(0) \beta^{-}(0), \quad i \in \mathcal{I}^{(0)}(p),
$$

the shift commuting map $\eta_{n}$ is to map a point $p \in P_{n}^{(0,1, r, \beta))}$ to the point $q \in P_{n}^{\circ}(F)$ that is given by

$$
q_{(i-n, i]}=\beta^{-}(0) \beta^{-} \beta^{-}(1) f(p) \beta^{-}(0) \beta^{-}(0), \quad i \in \mathcal{I}^{(0)}(p) .
$$

For a point $q \in \eta_{n}\left(P_{n}^{(0,1, r, \beta)}\right)$,

$$
q_{(i-n, i]} \in \mathcal{C}(0)^{*} \beta^{-}(0) \beta^{-}(0) \beta^{-}(0) \beta^{-} \beta^{-}(1), \quad i \in \mathcal{I}^{(1)}(q),
$$

and with the word $a(q) \in \mathcal{C}(0)^{*}$, that is given by writing

$$
q_{(i-n, i]}=a(q) \beta^{-}(0) \beta^{-} \beta^{-}(1), \quad i \in \mathcal{I}^{(0)}(q),
$$

a point $p \in P_{n}^{(0,1, r, \beta)}$ can be reconstructed from its image $q$ under $\eta_{n}$ as the point in $P_{n}^{\circ}(F)$ that is given by

$$
p_{(i-n, i]}=a(q) \beta^{+}(0) \beta^{-}(0) \beta^{-}(0) \beta^{+}(0), \quad i \in \mathcal{I}^{(0)}(q) .
$$

We note that

$$
\left(v_{0}\left(\lambda\left(\eta(p)_{[0, n)}\right)\right), v_{1}\left(\lambda\left(\eta(p)_{[0, n)}\right)\right)\right)=(3,1), \quad p \in P_{n}^{(0,1, r, \beta)} .
$$

Let $P_{n}^{(0,1, r, 1)}$ be the set of points

$$
p \in P_{n}^{\circ}\left(\alpha^{-}(0)\right) \backslash\left(P^{(1)} \cup P_{n}^{(\beta)} \cup P_{n}^{(\beta, 0)} \cup P_{n}^{(0,2)}\right)
$$

such that

$$
p_{(i-n, i]} \in \beta^{-}(0) \beta^{-}(0) \beta^{+}(0) \mathcal{C}(0)^{*} \beta^{+}(0) \beta^{-}(0), \quad i \in \mathcal{I}^{(0)}(p) .
$$

With the word $f(p) \in \beta^{-}(0) \beta^{+}(0) \mathcal{C}(0)^{*} \beta^{-}(0)$, that is given by writing

$$
p_{(i-n, i]}=\beta^{-}(0) \beta^{-}(0) \beta^{+}(0) f(p) \beta^{+}(0) \beta^{-}(0), \quad i \in \mathcal{I}^{(0)}(p),
$$


the shift commuting map $\eta_{n}$ is to map a point $p \in P_{n}^{(0,1, r, 1))}$ to the point $q \in P_{n}^{\circ}(F)$ that is given by

$$
q_{(i-n, i]}=\beta^{-}(1) \beta^{-} \Psi^{-1}(f(p)) \beta^{-}(1) \beta^{-}(0) \beta^{-} \quad i \in \mathcal{I}^{(0)}(p) .
$$

For a point $q \in \eta_{n}\left(P_{n}^{(0,1, r, 1)}\right)$,

$$
q_{(i-n, i]} \in \beta^{-} \beta^{-}(1) \beta^{-} \mathcal{C}(0)^{*} \beta^{-}(1) \beta^{-}(0), \quad i \in \mathcal{I}^{(0)}(q),
$$

and with the word $a(q) \in \mathcal{C}(0)^{*}$, that is given by writing

$$
q_{(i-n, i]}=\beta^{-} \beta^{-}(1) a(q) \beta^{-}(0), \quad i \in \mathcal{I}^{(0)}(q),
$$

a point $p \in P_{n}^{(0,1, r, \beta))}$ can be reconstructed from its image $q$ under $\eta_{n}$ as the point in $P_{n}^{\circ}(F)$ that is given by

$$
p_{(i-n, i]}=\beta^{-} \beta^{-}(1) \beta^{-} \Psi(a(q)) \beta^{-}(1) \beta^{-}(0), \quad i \in \mathcal{I}^{(0)}(q) .
$$

We note that

$$
\left(v_{0}\left(\lambda\left(\eta(p)_{[0, n)}\right)\right), v_{1}\left(\lambda\left(\eta(p)_{[0, n)}\right)\right)\right)=(1,2), \quad p \in P_{n}^{(0,1, r, 0)} .
$$

An inspection of the definition of $P_{n}^{(0,1, r, 1)}$ shows that we have produced a partition

$$
\begin{aligned}
P_{n}^{\circ}\left(\alpha_{-}(0)\right)= & P_{n}^{(1)} \cup P_{n}^{(\beta, \beta)} \cup P_{n}^{(\beta)} \cup P_{n}^{(\beta, 0)} \cup P^{(0,2)} \\
& \cup P_{n}^{(0,1, l)} \cup P_{n}^{(0,1, m)} \cup P_{n}^{(0,1, r)} \cup P_{n}^{(0,1, r, \beta)} \cup P_{n}^{(0,1, r, 0)} .
\end{aligned}
$$

The points of $\eta_{n}\left(P_{n}^{(1)}\right)$ are the only ones in $\eta_{n}\left(P_{n}^{\circ}\left(\alpha_{-}(0)\right)\right.$ in which there appears openly a word in $\mathcal{B}(1.1)$. In the points of $\eta_{n}\left(P_{n}^{(\beta)} \cup P_{n}^{(0,2)}\right)$, the word $\beta^{-} \beta^{-}(1)$ does not appear openly, whereas, in the words of $\eta_{n}\left(P_{n}^{(0,1, l)} \cup P_{n}^{(0,1, m)} \cup P_{n}^{(0,1, r)} \cup P_{n}^{(0,1, r, \beta)} \cup P_{n}^{(0,1, r, 0)}\right)$, this word does appear openly. In the points of $\eta_{n}\left(P_{n}^{(\beta, 0)}\right)$, the word $\beta^{-} \beta^{-}(1) \beta^{-}(0)$ appears openly, whereas, in the points of $\eta_{n}\left(P_{n}^{(0,1, l)} \cup P_{n}^{(0,1, m)} \cup P_{n}^{(0,1, r)}\right)$, this word does not appear openly. However, in the points of $\eta_{n}\left(P_{n}^{(0,1, l)} \cup P_{n}^{(0,1, m)} \cup P_{n}^{(0,1, r)}\right)$, the word $\beta^{-} \beta^{-}(1)$ does appear openly. The points of $\eta_{n}\left(P_{n}^{(0,1, r, \beta)}\right)$ are the only ones in $\eta_{n}\left(P_{n}^{\circ}\left(\alpha_{-}(0)\right)\right.$ in which there appears openly a word in $\left(\mathcal{C}^{*} \backslash \mathcal{C}(0)^{*}\right) \beta^{-}(0)$, and the points of $\eta_{n}\left(P_{n}^{(0,1, r, 1)}\right)$ are the only ones in $\eta_{n}\left(P_{n}^{\circ}\left(\alpha_{-}(0)\right)\right.$ in which there appears openly a word in $\beta^{-} \beta^{-}(1) \mathcal{C}(0)^{*} \mathcal{B}(1) \mathcal{C}(0)^{*}$.

From these observations and from $(1),(\beta),(\beta .0),(0.2)$, and $(0.1 .1),(0.1 . \mathrm{m}),(0.1 . \mathrm{r})$, (0.1.r. $\beta),(0.1 . r .0)$ it follows that the images under $\eta_{n}$ of the sets in the partition (P.0) are disjoint. Also, $\eta_{n}\left(P_{n}^{\circ}\left(\alpha_{-}(0)\right)\right) \cap P_{n}^{\circ}\left(\alpha_{-}(0)\right)=\emptyset$. We have shown that

$$
\operatorname{card} \mathcal{O}_{n}\left(\alpha^{-}(0)\right) \leq \operatorname{card}\left(\bigcup_{\tilde{\mu} \in \mathcal{M} \backslash\left\{\alpha^{-}(0)\right\}} \mathcal{O}_{n}\left(\tilde{\mu}^{-}\right)\right)
$$

Apply Lemma (c). 


\section{The multiplier $\alpha(1)$}

Proposition M1. The multiplier $\alpha(1)$ is exceptional only at period two.

Proof. We construct shift commuting injections

$$
\eta_{n}: P_{n}^{\circ}\left(\alpha_{-}(1)\right) \rightarrow \bigcup_{\tilde{\mu} \in \mathcal{M} \backslash\left\{\alpha_{-}(1)\right\}} P_{n}^{\circ}\left(\tilde{\mu}^{-}\right), \quad n>2 .
$$

Let $n>2$. Denote by $P_{n}^{(1)}$ the set of $p \in P_{n}^{\circ}\left(\alpha^{-}(1)\right)$ such that the word $\beta^{-} \beta^{-}(1)$ appears openly in $p$. The shift commuting map $\eta_{n}$ is to map a point $p \in P_{n}^{(1)}$ to the point $q \in P_{n}(F)$ that is obtained by replacing, in $p$, the word $\beta^{-} \beta^{-}(1)$, when it appears openly in $p$, by the word $\beta^{-}(0) \beta^{-}(0)$. A point $p \in P_{n}^{(1)}$ can be reconstructed from its image $q$ under $\eta_{n}$ by replacing, in $q$, every word $\beta^{-}(0)^{2 K}$, that appears in $q$ openly and that is neither preceded nor followed in $q$ by an open appearance of the symbol $\beta^{-}(0)$, by the word $\left(\beta^{-} \beta^{-}(1)\right)^{K}, K \in \mathbb{N}$.

Denote by $P_{n}^{(2)}$ the set of

$$
p \in P_{n}^{\circ}\left(\alpha^{-}(1)\right) \backslash P_{n}^{(1)}
$$

such that words in $\beta^{-} \mathcal{C}^{\circ}(1)^{*} \beta^{-}(1)$ appear openly in $p$ at least twice during a period. Denote by $\mathcal{J}(p)$, for $p \in P_{n}^{(2)}$, the set of indices $j \in \mathcal{I}^{(1)}(p)$ such that the word $p_{(n-j, n]}$ is lexicographically the smallest one among the words $p_{(n-i, n]}, i \in \mathcal{I}^{(1)}(p)$. With the word $f^{\circ}(p) \in \mathcal{C}^{\circ}(1)^{*}$, that is given by writing the word in $\beta^{-}\left(\mathcal{C}^{\circ}(1)^{*} \backslash\{\epsilon\}\right) \beta^{-}(1)$ that appears in $p$ at an index in $\mathcal{J}(p)$ as

$$
\beta^{-} f^{\circ}(p) \beta^{-}(1),
$$

the shift commuting map $\eta_{n}$ is to map a point $p \in P_{n}^{(2)}$ to the point $q \in P_{n}(F)$ that is obtained by replacing each of the words in the point $p$ (a), that appear in $p$ at an index in $\mathcal{J}(p)$, by the word

$$
\beta^{-}(0) \Psi\left(f^{\circ}(p)(p)\right) \beta^{-}(0) .
$$

With the word $f(q) \in \mathcal{C}(0)^{*}$ such that the word $\beta^{-}(0) f(q) \beta^{-}(0)$ appears openly in $q$, the point $p$ can be reconstructed from its image $q$ under $\eta_{n}$ by replacing, in $q$, the word

$$
\beta^{-}(0) f(q) \beta^{-}(0),
$$

when it appears openly in $q$, by the word

$$
\beta^{-} \Psi^{-1}(f(q)) \beta^{-}(1) .
$$

Denote by $P_{n}^{(3)}$ the set of

$$
p \in P_{n}^{\circ}\left(\alpha^{-}(1)\right) \backslash\left(P_{n}^{(1)} \cup P_{n}^{(2)}\right)
$$

such that

$$
p_{(i-n, i]} \in\left(\mathcal{C}^{*} \backslash\{\epsilon\}\right) \beta^{-} \mathcal{C}^{\circ}(1)^{*} \beta^{-}(1)
$$

With the words

$$
f(p) \in \mathcal{C}^{*}, \quad f^{\circ}(p) \in \mathcal{C}^{\circ}(1)^{*},
$$

that are given by writing

$$
p_{(i-n, i]}=f(p) \beta^{-} f^{\circ}(p) \beta^{-}(1)
$$


and denoting by $\Psi^{\prime}(c)$ the word that is obtained by removing from the word $\Psi\left(f^{\circ}(p)\right)$ its last symbol, the shift commuting map $\psi_{n}$ is to map a point $p \in P_{n}^{(3)}$ to the point $q \in P_{n}^{\circ}(F)$ that is given by

$$
q_{(i-n, i]}=f(p) \beta^{-}(0) \Psi^{\prime}\left(f^{\circ}(p)\right) \beta^{-} \beta^{-}(1), \quad i \in \mathcal{I}^{(1)}(q) .
$$

With the words

$$
f(q) \in \mathcal{C}^{*} \backslash\{\epsilon\}, \quad f^{\prime}(q) \in \mathcal{C}(0)^{*} \beta^{-}(0) \mathcal{C}(0)^{*},
$$

that are given by writing

$$
q_{(i-n, i]}=f(q) \beta^{-}(0) f^{\prime}(q) \beta^{-} \beta^{-}(1), \quad i \in \mathcal{I}^{(1)}(q),
$$

the point $p$ can be reconstructed from its image $q$ under $\eta_{n}$ as the point that is given by

$$
p_{(i-n, i]}=f(q) \beta^{-} \Psi^{-1}\left(f^{\prime}(q) \beta^{-}(0)\right) \beta^{-}(1), \quad i \in \mathcal{I}^{(1)}(q) .
$$

Set

$$
P_{n}^{(4)}=P_{n}^{\circ}\left(\alpha^{-}(1)\right) \backslash\left(P_{n}^{(1)} \cup P_{n}^{(2)} \cup P_{n}^{(3)}\right) .
$$

One has

$$
p_{(i-n, i]} \in \beta^{-}(1) \beta^{-} \mathcal{C}^{\circ}(1)^{*}, \quad i \in \mathcal{I}^{(1)}(p), \quad p \in P_{n}^{(4)} .
$$

With the words

$$
c(p) \in \mathcal{C}^{\circ}(1), \quad f^{\circ}(p) \in \mathcal{C}^{\circ}(1)^{*},
$$

that are given by writing

$$
p_{(i-n, i]}=\beta^{-}(1) \beta^{-} c(p) f^{\circ}(p), \quad i \in \mathcal{I}^{(1)}(p),
$$

the shift commuting map $\eta_{n}$ is to map a point $p \in P_{n}^{(4)}$ to the point $q \in P_{n}(F)$ that is given by

$$
q_{(i-n, i]}=\beta^{-}(1) \Phi_{0}(\Psi(c(p))) \Psi\left(f^{\circ}(p)\right) \beta^{-}, \quad i \in \mathcal{I}^{(1)}(p) .
$$

With the words

$$
b(q) \in \mathcal{B}(0,0), \quad g(q) \in \mathcal{C}(0)^{*},
$$

that are given by writing

$$
q_{(i-n, i]}=\beta^{-}(1) b(q) g(q) \beta^{-}, \quad i \in \mathcal{I}^{(1)}(q),
$$

the point $p$ can be reconstructed from its image $q$ under $\eta_{n}$ as the point that is given by

$$
p_{(i-n, i]}=\beta^{-}(1) \beta^{-} \Psi^{-1}\left(\Phi_{0}^{-1}(b(q))\right) \Psi^{-1}(g(q)), \quad i \in \mathcal{I}^{(1)}(q) .
$$

We have produced a partition

$$
P_{n}^{\circ}\left(\alpha_{-}(1)\right)=\bigcup_{1 \leq l \leq 4} P_{n}^{(l)} .
$$

In the points in $\eta_{n}\left(P_{n}^{(1)}\right)$, the word $\beta^{-}(0) \beta^{-}(0)$ appears openly, the word $\beta^{-} \beta^{-}(1)$ does not appear openly and, in the points in $\eta_{n}\left(P_{n}^{(2)}\right)$, neither the word $\beta^{-}(0) \beta^{-}(0)$ nor the 
word $\beta^{-} \beta^{-}(1)$ appear openly. In the points in $\eta_{n}\left(P_{n}^{(3)}\right)$ and $\eta_{n}\left(P_{n}^{(4)}\right)$, the word $\beta^{-} \beta^{-}(1)$ appears openly. Also,

$$
\begin{gathered}
\left(\mathcal{I}^{(1)}(q)+1\right) \cap \mathcal{I}^{(0)}(q)=\emptyset, \quad q \in \eta_{n}\left(P_{n}^{(3)}\right), \\
\mathcal{I}^{(1)}(q)+1 \subset \mathcal{I}^{(0)}, \quad q \in \eta_{n}\left(P_{n}^{(4)}\right) .
\end{gathered}
$$

From these observations it follows that the images under $\eta_{n}$ of the sets of the partition (P.1) are disjoint.

We have shown that

$$
\operatorname{card}\left(\mathcal{O}_{n}\left(\alpha^{-}(0)\right)\right) \leq \operatorname{card}\left(\bigcup_{\tilde{\mu} \in \mathcal{M} \backslash\{\alpha(1)\}} \mathcal{O}_{n}\left(\tilde{\mu}_{-}\right)\right) .
$$

Apply Lemma (c).

\section{REFERENCES}

[BP] J. Berstel and D. Perrin. The origins of combinatorics on words. European J. Combin. 28 (2007), 996-1022.

[BPR] J. Berstel, D. Perrin and Ch. Reutenauer. Codes and Automata. Cambridge University Press, Cambridge, 2010.

[HI] T. Hamachi and K. Inoue. Embeddings of shifts of finite type into the Dyck shift. Monatsh. Math. 145 (2005), 107-129.

[HIK] T. Hamachi, K. Inoue and W. Krieger. Subsystems of finite type and semigroup invariants of subshifts. J. Reine Angew. Math. 632 (2009), 37-61.

[HK] T. Hamachi and W. Krieger. A construction of subshifts and a class of semigroups. Preprint, 2013, arXiv:1303.4158 [math.DS].

[Ki] B. P. Kitchens. Symbolic Dynamics. Springer, New York, 1998.

[KM] W. Krieger and K. Matsumoto. Zeta functions and topological entropy of the Markov-Dyck shifts. Münster J. Math. 4 (2011), 171-184.

[Kr1] W. Krieger. On the uniqueness of the equilibrium state. Math. Syst. Theory 8 (1974), 97-104.

[Kr2] W. Krieger. On a syntactically defined invariant of symbolic dynamics. Ergod. Th. \& Dynam. Sys. 20 (2000), 501-516.

[LM] D. Lind and B. Marcus. An Introduction to Symbolic Dynamics and Coding. Cambridge University Press, Cambridge, 1995.

[M] K. Matsumoto. $C^{*}$-algebras arising from Dyck systems of topological Markov chains. Math. Scand. 109 (2011), 31-54.

[NP] M. Nivat and J.-F. Perrot. Une généralisation du monoîde bicyclique. C. R. Math. Acad. Sci. Paris 271 (1970), 824-827.

[P] D. Perrin. Algebraic combinatorics on words. Algebraic Combinatorics and Computer Science. Eds. H. Crapo and D. Senato. Springer, Berlin, Heidelberg, New York, 2001, pp. 391-430. 